\title{
Generalized Lagrangian Coherent Structures
}

\author{
Sanjeeva Balasuriya \\ School of Mathematical Sciences, University of Adelaide, Adelaide, SA 5005, Australia \\ Nicholas T. Ouellette \\ Department of Civil and Environmental Engineering, Stanford University, Stanford, CA \\ 94305, USA \\ Irina I. Rypina \\ Department of Physical Oceanography, Woods Hole Oceanographic Institution, Woods \\ Hole, MA 02543, USA
}

\begin{abstract}
The notion of a Lagrangian Coherent Structure (LCS) is by now well established as a way to capture transient coherent transport dynamics in unsteady and aperiodic fluid flows that are known over finite time. We show that the concept of an LCS can be generalized to capture coherence in other quantities of interest that are transported by, but not fully locked to, the fluid. Such quantities include those with dynamic, biological, chemical, or thermodynamic relevance, such as temperature, pollutant concentration, vorticity, kinetic energy, plankton density, and so on. We provide a conceptual framework for identifying the Generalized Lagrangian Coherent Structures (GLCSs) associated with such evolving quantities. We show how LCSs can be seen as a special case within this framework, and provide an overarching discussion of various methods for identifying LCSs. The utility of this more general viewpoint is highlighted through a variety of examples. We also show that although LCSs approximate GLCSs in certain limiting situations under restrictive assumptions on how the velocity field affects the additional quantities of interest, LCSs are not in general sufficient to describe their coherent transport.

Keywords: Lagrangian coherent structures, fluid flow, flow barriers, ocean eddies, atmospheric vortices
\end{abstract}




\section{Introduction}

The concept of a 'coherent structure' has a long history in fluid mechanics, both as a tool for qualitative flow visualization and understanding and as a basis for reduced-order modeling. Most traditional approaches to determining coherent structures take an Eulerian approach, considering single snapshots in time and typically analyzing the streamline structure. For steady flows, this streamline picture gives full information about how fluid particles travel for all times, since the streamlines remain the same forever and coincide with the trajectories of fluid particles. One can then take a classical dynamical-systems approach to analyzing the flow. In this framework, entities such as stagnation points (that is, zeros of the velocity field) and their associated stable and unstable manifolds (i.e., material curves that contain fluid parcels that approach a saddle-like hyperbolic stagnation point in forward of backward time) are crucial for separating space into regions in which the topological behavior of the flow is different [1]. The codimension1 (that is, one dimension less than the physical space) stable and unstable manifolds therefore can be thought of as important flow separators that demarcate regions of coherent fluid blobs. Separators might also be identified in time-periodic flows by defining a discrete-time Poincaré map [2] with no explicit time-variation. In addition to stable/unstable manifolds, families of nested KAM tori $[2,3]$, which enclose a region around an elliptic periodic point, represent another important type of coherent eddy-like objects. We note that the term "coherent" has no universally agreed-upon precise definition in fluid mechanics [4]; here, we will use it roughly to mean "sharing the same qualitative spatiotemporal behavior."

When the flow is unsteady with a more complicated non-periodic time dependence, Poincaré maps cannot be applied to remove the time variation, and the situation changes. In a series of seminal and influential papers, Haller and collaborators $[5,6,7]$ pointed out 18 years ago that when analyzing the coherence of fluid blobs in unsteady flows, purely Eulerian diagnostics based on velocities or instantaneous streamfunction contours may give misleading information ${ }^{1}$. This work gave birth to the field of Lagrangian Coherent Structures (LCSs), a term coined by Haller and Yuan [5], that has since seen extensive research $[9,10,11,1,12,13]$. The initial guiding observation that spurred this activity was that Eulerian entities such as nested closed stream-

\footnotetext{
${ }^{1}$ Short time diagnostics are possible in some instances; see [8].
} 
lines do not necessarily correspond to a Lagrangian vortex structure because, unlike in the steady situation, fluid particles are not confined to move along the instantaneously frozen streamfunction contours. Methods such as the Okubo-Weiss-Chong criterion $[14,15,16]$ and its various counterparts and extensions $[17,18,19,20]$ for separating the flow into rotating and stretching regions based on the instantaneous velocity field are similarly unable to capture the true Lagrangian motion.

Even in unsteady flows defined over infinite times, stable and unstable manifolds continue to be important flow separators, although they must be defined and interpreted somewhat differently. Rather than containing points that decay in forward or backward time to a stagnation point, they now contain points that decay to hyperbolic trajectories, which themselves move about in time. The identification of the hyperbolic trajectories formally requires the difficult testing of a definition of exponential dichotomies [21, $22,23]$, which is essentially a statement about exponential decay akin to what occurs along the stable and unstable manifolds of hyperbolic (saddlelike) stagnation points in steady flows ${ }^{2}$. In unsteady flows, the manifolds move with time and intersect in many ways $[1,25]$, resulting in complicated transport possibilities. The viewpoint is now Lagrangian, since it explicitly examines the flow of fluid particles according to an unsteady velocity field. Specifically, we consider $x \in \Omega \subseteq \mathbb{R}^{n}$, where $\mathbb{R}^{n}$ is our physical space. In typical fluids applications, $n=2$ or 3 . Fluid particle trajectories are solutions of

$$
\dot{x}=v(x, t),
$$

where $v(x, t)$ is the flow velocity at position $x$ and time $t$. We note that we will use simple letters - unadorned with vector symbols or indices - in our notation throughout for scalars, vectors or tensors, as is common in more mathematically-inclined work. The velocity in (1) is assumed known, or at least measured from experimental or numerical data, though potentially only at discrete locations in time and space. For time-periodic flows, there are methodologies to help determine stable and unstable manifolds, KAM tori, and the associated transport mechanisms between the coherent structures they distinguish $[26,27,28,29]$. Some of these techniques can be extended to flows with aperiodic time dependence under certain conditions [30, 31].

While time-periodic flows arise in some realistic situations (such as in

\footnotetext{
${ }^{2}$ See Appendix A in ref. [24] for more intuition.
} 
periodically-driven industrial flows), in many other situations $v$ has aperiodic time dependence and is only known via data over a finite time interval. Using exponential decay as a criterion becomes problematic, since any function of $t$ can be bounded by a term of the form $A e^{\lambda t}$. Thus stable and unstable manifolds, in the classical sense of ordinary differential equations theory, cannot be defined. Nevertheless, it is tempting to generalize these ideas to aperiodic, finite-time situations, and a number of less restrictive definitions have been proposed to identify the finite-time analogs of infinite-time stable and unstable manifolds and KAM tori in realistic fluid flows. These structures or, somewhat confusingly, the regions of fluid delineated by them, have come to be called Lagrangian Coherent Structures (LCSs). We will maintain both these understandings throughout this article, making the distinction when needed.

At this point, a multitude of diagnostic definitions, not all of them consistent with one another, exist to try to accomplish the goal of identifying LCSs (as will be discussed in more detail in Section 2). Some aim to take advantage of properties that one would expect for stable and unstable manifolds in flows with simpler time dependence that are known for infinite times. In Finite-Time Lyapunov Exponent (FTLE) approaches, for example, one seeks neighboring points that separate exponentially quickly at a rate anomalously larger than in nearby regions. This method uses the intuition gained from studying infinite-time flows that a fluid element placed on a stable manifold will in forward time stretch exponentially in the complementary direction, since it will eventually be influenced by the associated unstable manifold of the hyperbolic trajectory. Many other methods that define and locate LCSs have also been developed, as we survey. We refer to these techniques as 'diagnostic methods' for determining LCSs, but our primary discussion will be essentially independent of which method is used. The key point is whatever the definitions of LCSs, their formulation is typically (with a few exceptions, such as, for example, the burning manifolds of [32, 33, 34, 35, 36]) based solely on the Lagrangian trajectories associated with (1), and the goal is to identify fluid parcel groups that behave similarly, and are thus 'coherent.' This has been a rich area of study using both experimental [37, 38, 39, 40] and observational $[41,42,43,44,45,46,47,48,49,50,51,52,53]$ data.

In many situations of practical interest, fluid particle locations and mass transport are not the only quantities of interest. Rather, one often desires information about the coherence of other quantities that are impacted by the flow. Here, we shall refer to such quantities as co-evolving variables, with 
the understanding that they are scalar or vector fields in space which evolve with time following an equation of motion that depends on the velocity field but potentially contains other dynamics as well. A common example is the concentration of a tracer, such as a chemical pollutant or water vapor that is passively advected by the flow while also diffusing/decaying in time. While tracers may, to first approximation, be thought of as dynamically passive scalars that do not affect the fluid velocities, another class of examples are those of dynamically active scalar or vector variables, whose evolution directly affects the flow velocity. One such quantity is the vorticity, whose evolution is mutually dependent on $v$, as specified by the vorticity equation that can be derived from the Navier-Stokes equations $[54,55]$. The temperature might be thought of as either dynamically active (e.g., coupled to the velocity via the Boussinesq approximation in situations such as Rayleigh-Bénard convection [55]), or dynamically passive (e.g., carried by, but not affecting, the flow velocities $[56,44,45])$ depending on the context. In these examples, relevant "coherent" entities refer to regions of space characterized by the qualitatively similar behavior of the co-evolving variable, for example, regions containing similar tracer concentrations, or the codimension-1 boundaries between these regions, across which there are sharp tracer gradients. Another example where the use of a co-evolving variable would be relevant is in the situation of two different fluids in a microfluidic device, with the interface between them being thought of as the "coherent" object of interest that we would like to track. In all these cases, standard LCSs are unable to capture coherence of the relevant co-evolving variables, because they are based on (1) and have no additional information about the behavior of the co-evolving variable(s).

The way in which the co-evolving fields are affected by the fluid velocity $v$ depends on the physical, chemical, biological, or geophysical aspects governing their behavior. That is, there typically need to be other equations, which we shall term evolution equations, in addition to, or replacing, (1). In Section 3, we develop a conceptual framework to take these into account. We term the coherent structures obtained from the evolution equations Generalized Lagrangian Coherent Structures (GLCSs). To set the stage, we will need to understand LCSs - i.e., the entities pertaining to (1) without considering any additional evolution equations - and therefore we precede the GLCS development with Section 2, in which we provide a framework for LCSs. While our discussion is independent of the particular diagnostic method used for defining an LCS, we provide details on how several commonly used definitions for LCSs fit into this framework. We also discuss several broad issues related 
to LCSs and their validity, including differences between two or three dimensions, frame-independence of LCS methods, the time-variation of LCSs and whether they follow the flow. Thereafter, in Section 3 we develop a framework for GLCSs, and show how our GLCS definition includes LCS as a special case. Additionally, we discuss a variety of examples that fall within the GLCS framework. Relevant problems include evolution of tracers in fluid flows with or without diffusion, evolution of the boundaries between two immiscible fluids, modified velocities in a range of contexts (effective velocities for inertial particles, fluid velocity modification due to swimming organisms, front propagation velocity, stochastic perturbations), and the evolution of vorticity, potential vorticity and kinetic energy. We highlight that LCSs are not guaranteed to identify coherent structures of interest for the co-evolving variables in these examples. Several detailed case studies are presented in Section 4, including a passively advected pollutant in a double gyre, the flow interface between two miscible or immiscible fluids in a micro-channel mixer, the observed sea-surface temperature in the North Atlantic ocean, and a tracer advected by the satellite-derived oceanic velocities. In each case we compare and contrast the relevant GLCSs with the LCSs. Typically, these do not coincide, except in some limiting situations. We conclude in Section 5 with a summary, highlighting the fact that different LCS methods offer different results, and that these are not always related to a particular GLCS.

\section{Lagrangian Coherent Structures (LCSs)}

\subsection{Framework for LCSs}

LCSs are coherent entities that are associated with, and should be evident from viewing trajectories of fluid parcels obeying, Eq. (1). Intuitively, LCSs ought to be generalizations of the easily identifiable coherent flow structures, such as vortices/eddies, jets, and stable and unstable manifolds in steady, two-dimensional, infinite-time flow. Exactly which properties are used to make such a generalization leads to different definitions of LCSs. Here, we will abstractly represent LCSs in a way that is consistent with any definition. What is important here is that the LCS is associated only with trajectories of (1), with no other quantity (e.g., temperature, pollutant concentration, fish larvae) being considered as a co-evolving variable of interest.

To make our discussion compatible with the finite-time limitations of data, we assume that the flow (1) is known within a finite-time interval 
$[-T, T]$; such centering can be done without loss of generality for any finitetime interval. We want to define LCSs as subsets of the spatial domain $\Omega$ at time $t \in(-T, T)$. These subsets may have the same or smaller dimensions than $\Omega$. In addition to the current (or starting) time $t$ at which the LCS is to be defined, one needs to specify the time interval over which one seeks coherence of fluid particle trajectories. For example, the region within the core of a transient eddy would only be coherent over the lifetime of an eddy, but not over a much longer time interval. Thus, in addition to the current time $t$, we will also introduce a reference (or final) time $\tau \in[-T, T]$ in relation to which LCS structures at time $t$ are found. The LCSs are then computed using velocity data from the interval $[t, \tau]$ if $\tau>t$ or $[\tau, t]$ if $\tau<t$, and the coherence is specific to this finite-time interval.

As the first step towards identifying LCSs, for a point (or initial position) $x$ in $\Omega$, we define the flow map from time $t$ to $\tau, F_{t}^{\tau}(x)$, by following the evolution of $x$ under (1) from time $t$ to $\tau$. In other words, the flow operator $F_{t}^{\tau}(x)$ is the solution to the Lagrangian form of (1) that advects the initial position $x$ at time $t$ to its final position at time $\tau$, i.e.,

$$
\frac{\partial F_{t}^{\xi}(x)}{\partial \xi}=v\left(F_{t}^{\xi}(x), \xi\right) \quad, \quad F_{t}^{t}(x)=x
$$

solved up to time $\xi=\tau$. Equivalently,

$$
F_{t}^{\tau}(x)=x+\int_{t}^{\tau} v\left(F_{t}^{\xi}(x), \xi\right) \mathrm{d} \xi
$$

The flow operator can be applied in both forward and backward time (because the subscript might be a larger value that the superscript), since the flow of the ordinary differential equation (1) is reversible. For any deterministic flow, it additionally satisfies the condition $F_{\tau}^{t}\left(F_{t}^{\tau}(x)\right)=x$ because following the flow from $t$ to $\tau$, and subsequently from $\tau$ back to $t$, takes one back to the original point $x$. In other words, the flow operator must be invertible, i.e., $\left[F_{\tau}^{t}\right]^{-1}=F_{t}^{\tau}$.

The conditions that are usually required for LCS computation, are that $F_{t}^{\tau}: \Omega \rightarrow \Omega$ is a well-defined, invertible and smooth map. This, in turn, requires that the velocity field $v$ has continuous first derivatives in both space and time, ensuring that trajectories are uniquely defined for all initial conditions throughout $\Omega$. This is stated in Definition $1^{3}$.

\footnotetext{
${ }^{3}$ Some relaxations of these conditions are possible $[57,30]$. The typical existence and
} 
Definition 1. The Lagrangian evolution (1) is to be considered for $x \in \Omega$, where $\Omega$ is some open connected subset of $\mathbb{R}^{n}$ (typically, $n=2$ or 3 ), and time is in $[-T, T]$. Let $v: \Omega \times[-T, T] \rightarrow \mathbb{R}^{n}$, such that $v \in \mathrm{C}^{1}(\Omega \times(-T, T))$.

A possible criterion for identifying LCSs might be that the LCS is a subset $S \in \Omega$ chosen at time $t$, such that $F_{t}^{\tau}(S)$ is similar to $S$ in that both consist of connected regions whose boundaries are nonfilamented. This could be an attempt to identify a coherent core of a vortex. Another possible criterion might be that $F_{t}^{\tau}(S)$ stretches much more, or attracts much more strongly, than $F_{t}^{\tau}(\tilde{S})$ for any set $\tilde{S}$ chosen near $S$. This would be an attempt to capture codimension- 1 entities corresponding to finite-time analogues of unstable manifolds [9]. The key point is that some criterion must be chosen to identify the LCS, with different criteria potentially yielding different LCSs adapted to the specific issue of interest.

We can represent any such criteria by a procedure or operator, $\mathcal{P}$, that is being applied to the flow map, i.e.,

$$
\operatorname{LCS}(t, \tau):=\mathcal{P}\left(F_{t}^{\tau}(\Omega)\right) .
$$

The resulting quantity, $\operatorname{LCS}(t, \tau)$, identifies a subset of $\Omega$ at time $t$ according to criterion $\mathcal{P}$ applied to the vector field $F_{t}^{\tau}$ (which is defined on $\Omega$ ). The first argument in the notation for LCS stands for the time instance in which the LCS is being identified, while the second is the reference time associated with the end of the finite-time flow interval that is being used. The 'Lagrangian' aspect of LCSs is highlighted by the fact that the identified sets are associated with tagging particles at time $t$, and uses a field associated with the Lagrangian evolution of these particles by (1); in other words, this field is represented in Lagrangian rather than Eulerian coordinates. Note that the quantity $F_{t}^{\tau}(\Omega)$ in the r.h.s. of (3) generally needs to be calculated for a dense set of initial conditions $x$ spanning the domain $\Omega$. Moreover all initial conditions $x \in \Omega$ have equal consideration; there is no prejudicing of certain $x \mathrm{~s}$ in

uniqueness theorem for solutions of ordinary differential equations such as (1) require that the vector field $v$ be Lipschitz-continuous in space and bounded in time, which are weaker conditions than in Definition 1. However, we retain the continuous first-derivative condition for ease of understanding; this is indeed what is presented in [2]. Moreover, our stronger condition helps make the flow map differentiable, which is often required in LCS methods. We note that fully turbulent flows do not normally satisfy Definition 1, since the velocity field is only Hölder-continuous [58]. 
computing $F_{t}^{\tau}(x)$. The procedure $\mathcal{P}$ is then applied - this typically consists of determining a field resulting from $F_{t}^{\tau}(\Omega)$, and then using some criterion on this field - to identify those trajectories, or, more specifically, the time- $t$ position of those trajectories, which satisfy the specified criteria. The set of the obtained initial positions forms the LCS. It must be emphasized that LCSs in (3) are conjoined with the procedure $\mathcal{P}$, so speaking of an LCS without stating how it was defined is meaningless.

\subsection{LCS diagnostic methods}

In this section, we discuss several operators $\mathcal{P}$ in (3) that are associated with commonly used LCS definitions. Our review is not exhaustive. Our purpose is to motivate the generalization of the concept of LCSs, and we focus below only on methods that have seen the most activity and application. There are other LCSs methods that are not discussed here, and the ordering we choose is based on ease of description according to our framework.

(a) Averages along trajectories: There are a variety of procedures that rely on computing the quantity

$$
A(t, \tau, x)=\frac{1}{|t-\tau|} \int_{t}^{\tau} g\left(b\left(F_{t}^{\xi}(x), \xi\right)\right) \mathrm{d} \xi,
$$

for some function $g$ and some observable $b$, and then applying an operator to extract some special features of the $A$-field. Quantity $A$ is an average along Lagrangian trajectories $[59,60,61,62,63]$, and some authors term these methods 'Lagrangian descriptors.' The choice of $g$ and $b$ determines what exactly is being averaged. A common choice is $b=v$, the velocity field itself $[61,62,60,59,50]$. If additionally $g(z)=|z|$, one is averaging a particle's speed along a trajectory, and hence $A(t, \tau, x)$ represents the path length of a trajectory $[60,59,50]$. If instead $g(z)=z$ then $A(t, \tau, x)=\left(F_{t}^{\tau}(x)-x\right) /|t-\tau|$, which defines an average velocity $[61,62]$. Other options include the combined function $g \circ b$ being the instantaneous eigenvector field of the variational equation [41], or a set of indicator functions over spatial boxes in order to define trajectory's "correlation dimension" or "ergodicity defect" $[64,50]$. Averages of the form (4) are also relevant to determining dispersion statistics [65], which are expected to be different in different coherent regions of a fluid. An intriguing method proposed 
recently is associated with setting

$$
g \circ b=|t-\tau|\left|\omega\left(F_{t}^{\xi}(x), \xi\right)-\bar{\omega}(\xi)\right| ; \omega=\nabla \times v,
$$

in which $\omega$ is the vorticity and $\bar{\omega}$ is its average over the entire domain at each time. Then, $A$ is the Lagrangian Averaged Vorticity Deviation (LAVD) proposed by Haller et al [63] which-rather than specified in an ad hoc fashion - was derived in seeking a frame-independent quantification of rotation. All of these methods use an intuition that trajectories in different (same) coherent regions will have qualitatively different (similar) behavior and thus different (similar) values of the averaged quantities. The procedure $\mathcal{P}$ consists of computing (4), treating it as a scalar or vector field on $\Omega$, and then extracting ridges (that is, locally maximizing curves), valleys (locally mininizing curves), curves of highest gradient of the $A(t, \tau, x)$-field or, in the case of the LAVD, outermost convex closed contours; these serve as proxies for the boundaries between coherently moving regions of fluid [59, 60, 61, 62, 63, 50], or looking for the coherent regions themselves as areas characterized by plateaus of similar (almost constant) $A$ values. While there is not always a rigorous way to justify a relationship between 'coherence' and ridges/plateaus of $A$, an interesting correspondence is found in some flows.

(b) Asymptotic approximations: The role of stable/unstable manifolds as flow barriers between coherent structures in steady, infinite-time flows has been well-understood for decades (see [1]). Thus, $\mathcal{P}$ could be a procedure for theoretically determining analytic approximations to the stable and unstable manifolds via artificially extending the flow (1) from $[-T, T]$ to $\mathbb{R}$, and then looking at restrictions of these entities to the actual time interval $[-T, T][66,31,67,1]$. A reasonable extension for nearly steady flows is to take the time-averaged velocity field from the given data in $[-T, T][66]$. Subsequently, the reference time is $\tau= \pm \infty$ for the artificial flow, with the sign chosen depending on whether one is looking for stable or unstable manifolds.

(c) Variational LCSs: The variational method defines hyperbolic LCSs as the locally most repelling or attracting curves (or surfaces in three dimensions) that are materially advected under the flow (1) from a 
time $t$ to $\tau$ [9]. The procedure for their determination involves the eigenvalues and eigenvectors of the Cauchy-Green strain tensor $[9,68]$

$$
C_{t}^{\tau}(x):=\left[D F_{t}^{\tau}(x)\right]^{\top}\left[D F_{t}^{\tau}(x)\right],
$$

in which $D F_{t}^{\tau}(x)$ is the Jacobian derivative matrix of the flow map $F_{t}^{\tau}(x)$. The operator $\mathcal{P}$ uses spatial fields of these eigenvalues and eigenvectors to identify curves (in 2D [69, 68, 70]) or surfaces (in 3D $[71,72])$ of extremal attraction or repulsion in the time-slice $t$, with respect to the time evolution from $t$ to $\tau$. The variational methods also exist for elliptic and parabolic structures [9], and the excellent review article [9] is recommended for readers requiring additional information on how to compute variational LCSs.

(d) Finite-Time Lyapunov Exponents (FTLEs): Possibly the most frequently used method for identifying LCSs is based on the FTLE field $[73,9,74,75,9,76,77,78,79]$,

$$
\operatorname{FTLE}(t, \tau, x)=\frac{1}{|t-\tau|} \ln \max _{|z| \neq 0} \frac{\left|D F_{t}^{\tau}(x) z\right|}{|z|} .
$$

The Jacobian matrix derivative of $F, D F$, encapsulates leading-order behavior and represents the first term in the Taylor expansion of the r.h.s. of (1) near $x$. Using the analysis of matrix norms [80], a computationally efficient way for evaluating (7) directly from grids of Lagrangian trajectories can be written in terms of the Cauchy-Green tensor (6) as

$$
\operatorname{FTLE}(t, \tau, x)=\frac{1}{|t-\tau|} \ln \sqrt{\lambda(t, \tau, x)},
$$

where $\lambda(t, \tau, x)$ is the largest eigenvalue of $C_{t}^{\tau}(x)$. The FTLE characterizes the maximum exponential separation rate between a trajectory and its close neighbors in all directions, suitably normalized and averaged. FTLEs can be computed in forward and backward time, depending on the sign of $t-\tau$. The most common implementation takes $\tau=t+t_{w}$ (forward-time) or $t-t_{w}$ (backward) for a fixed windowing time $t_{w}$. The dependence on $t_{w}$ highlights the little-mentioned fact that pictures of FTLE fields have an inherent time-scale (i.e., $t_{w}$ ) associated with them. The procedure $\mathcal{P}$ for defining LCSs consists of computing the forwardor backward-time FTLE field, and then extracting strong maximizing 
ridges, motivated by the fact that the exponential decay rates associated with stable and unstable manifolds are captured by (7) respectively as $\tau \rightarrow \infty$ or $-\infty$ for infinite-time flows. Note, however, that although maximizing ridges of FTLEs correspond to attracting or repelling manifold-type structures in most fluid flows, unexpected results are possible: the maximum stretching is along and not perpendicular to the ridge $[9,81]$, a trough (rather than a ridge) of the backward-time FTLE field is associated with an unstable manifold [81, 82], or a stable manifold is not identifiable as a ridge of a forward-time FTLE field $[81,82]$.

The Finite-Size Lyapunov Exponent (FSLE) is closely related to the FTLE, but unlike in (7) where the displacement $(D F) z$ of nearby particles is assessed over a fixed time interval of length $t-\tau$, the computation is performed until a fixed separation between a trajectory and its nearby neighbors is achieved, and the relevant time $\tau$ is inserted into (7) $[83,84,85,86]$. FTLE and FSLE methods have seen wide usage in both experimental $[37,38,39,40]$ and observational $[42,43,47,83,84,85,46,49,50,51]$ contexts.

(e) Transfer-operator methods: The transfer or Perron-Frobenius operator $\Phi_{t}^{\tau}$ encodes how a density distribution at time $t$ is mapped to a distribution at time $\tau$ after advection by the flow (1), i.e., after applying the flow map (3) to passively advect the initial density distribution. A discretized approximation for $\Phi_{t}^{\tau}$ can be obtained by partitioning the domain into tiny cells, and numerically determining the probability that (1) transports particles from one cell to another. Sets which are nearly invariant under small perturbations [87] are associated with the singular-values/vectors of the transfer operator, i.e., the eigen-values/vectors of $\left(\Phi_{t}^{\tau}\right)^{\top} \Phi_{t}^{\tau}$. In the simplest application of transfer operator methods, the second-top singular vector (which assigns a value to each tiny cell, thereby approximating a scalar field on $\Omega$ ) is computed [88], and then partitioned according to a procedure $\mathcal{P}$ to form two 'almost invariant sets' which correspond to $\operatorname{LCS}(t, \tau)$ in our notation. Extending this partitioning idea in various ways, and/or utilizing higher-order singular vectors, is the basis of the transfer operator method for obtaining coherent structures [89, 90, 88, 91].

(f) Braiding: In two-dimensional flows, a measure of trajectory intermin- 
gling is quantified by the topological entropy that can be computed by keeping track of how trajectories wind around each other, i.e., how they braid. The method starts with computing Lagrangian trajectories for a number of initial positions, i.e., $F_{t}^{\tau}\left(x_{i}\right), i=1,2, \cdots, N$. The procedure $\mathcal{P}$ then consists of computing the topological entropy associated with combinations of these trajectories [92, 93, 94, 95], and then separating trajectories into groups which have nearly identical entropy. Numerical application of this method requires that $N$ be not large $(\sim \mathcal{O}(50))$ to be able to address all possible combinations of trajectories.

(g) Clustering: These methods apply clustering techniques from the machinelearning community, such as fuzzy clustering algorithms [96], spectral clustering [97], or graph-based partitioning [98, 99], to identify clusters of similarly behaving Lagrangian trajectories. In a way, one may argue that all of the methods described above also involve some sort of clustering, in that they all start with computing large number of trajectories and then separate these trajectories into sets or clusters that have similar behavior according to some criterion.

(h) Encounter volume: The encounter volume method [100] conceptualizes mixing as an irreversible exchange of properties between different fluid masses, and quantifies this by how often fluid parcels come in contact with each other. An objective measure of encounters between fluid elements, the encounter volume $V\left(t, \tau, x_{0}\right)$, can then be introduced as the combined volume of all the fluid elements that pass within a radius $R$ of the reference fluid element (which is at $x_{0}$ at time $t$, but is traveling with the flow) over a time interval $[t, \tau]$. To compute this, a grid of particles $x_{1}, x_{2}, \cdots, x_{K}$ is seeded in $\Omega$ at the initial time $t$, the particles are advected by the flow to time $\tau$, and the encounter number is counted for each particle using

$$
N\left(t, \tau, x_{i}\right)=\sum_{k=1, k \neq i}^{K} I\left(\min _{\xi \in[t, \tau]}\left|F_{t}^{\xi}\left(x_{k}\right)-F_{t}^{\xi}\left(x_{i}\right)\right| \leq R\right),
$$

with the indicator function $I=1$ if true and $I=0$ if false. The encounter volume

$$
V\left(t, \tau, x_{i}\right)=N\left(t, \tau, x_{i}\right) \Delta V
$$


can then be computed by attributing a little fluid element volume $\Delta V$ to each trajectory $(\Delta V$ is defined by the initial spacing between trajectories and is constant following any trajectory for incompressible flows). By performing this computation for each of the initial conditions $\left(x_{1}, x_{2}, \cdots x_{K}\right)$, a scalar field $V\left(t, \tau, x_{i}\right), i=1,2, \cdots, K$, can be defined on a discretized $\Omega$. Regions of space with large/small values of $V$ indicate domains of flow at time $t$ with enhanced/inhibited mixing potential, and are thus coherent in this sense. Since a large number of encounters happens in the vicinity of hyperbolic trajectories, and because manifolds serve as pathways that bring particles toward the hyperbolic trajectory in forward/backward time, maximizing ridges of the $V$-field computed in forward and backward time will approximate finite-time counterparts of the stable and unstable manifolds, respectively. On the other hand, $V$ would is small inside an eddy core, where encounters are limited to fluid parcels within the same core. Thus, the procedure $\mathcal{P}$ consists of estimating the encounter volume $V(t, \tau, x)$ and then identifying maximizing ridges (proxy manifolds) and minimizing plateaus (proxy eddy cores) of $V$. This method specifically targets the characterization of diffusive mixing [101].

(i) Other methods: In addition to what we have described above, many other methods have been proposed to find LCSs. These include locating sets that preserve their shape [102], methods that keep track of the maximum of an observable along trajectories instead of the average value as in trajectory averages methods (i.e., $\mathrm{L}^{\infty}$-norm is used instead of $\mathrm{L}^{1}$-norm in (4) [103]), finite-time entropy [104], curves of minimum flux [105], Oseledets spaces [66], ergodic quotient [106], patchiness [107], finite-time curvature [102, 108], and others [109].

The above list is non-exhaustive; other LCS identification methods have also been proposed, and many more continue to be developed. Our purpose is not to enter into the debate as to which diagnostic is most appropriate, better justified, most computationally attractive, most robust, most coherently follows the flow (1), and so on ${ }^{4}$. Rather, we wish to illustrate that in all of the definitions discussed above, LCSs are based purely on the trajectory

${ }^{4}$ For a recent article comparing the merits of a range of LCS methods, the reader is referred to $[110]$. 
information of (2), and thus capture coherence associated only with fluid trajectories of (1), independently of the coherence measure used. Coherence of co-evolving variables may be different, and LCSs are not guaranteed to reveal it. The need for methods that can capture coherence of something other than fluid trajectories have urged researchers to extend the concept of LCSs to some chemical and bio-physical applications by using a modified velocity field in $(1)[32,33,34,35,36,111,112,113,114]$. In the next section we present a unified notation of generalized LCSs that naturally incorporate these prior developments and are readily applicable to other co-evolving variables not considered in prior literature.

\subsection{Aspects of LCS diagnostics}

As a final step before introducing our generalization, we briefly summarize some additional issues that often arise in the discussions of LCS methods, as they will be relevant to our generalized LCSs as well.

1. Flow dimensionality: The most common application of the LCS methods is to two-dimensional flows, owing both to numerical and visualization ease and theoretical simplicity. Some methods, such as braiding or infinite-time asymptotic approximations, are explicitly restricted to two dimensions, with no obvious way to extend to three. Others, including FTLEs, trajectory averages, transfer operators, variational LCSs, clustering, and encounter volume, can and/or have been extended to three dimensions. Applications to three dimensions are less common because they are computationally expensive, rely on the accurate knowledge of the time-varying 3D velocity field that is challenging from both observational or modeling perspectives, and require advanced visualization of LCSs. As the LCS methods advance, in parallel with experimental ability to measure highly resolved 3D velocity fields [115], it is hoped that our ability to tackle three-dimensional unsteady flows will improve.

2. Time-parametrization: LCSs are often thought of as being parametrized by the time $t$ in which they are defined. However, $t$ is only the first argument of LCS in (3), and the presence of the second argument $\tau$ (the reference time) complicates this interpretation. Consider for example a standard forward-time FTLE implementation which uses the field $\operatorname{FTLE}\left(t, t+t_{w}, x\right)$ from (7) to determine $\operatorname{LCS}\left(t, t+t_{w}\right)$ for a fixed windowing time $t_{w}$. The idea of the LCSs being time-parametrized is 
disturbed here, because varying $t$ means that both the time-instance at which the LCS is computed, and the reference time are varied (see also [9] for more discussion on the issues with the often used 'slidingwindow' approach). A way to retain a consistent time-parametrization might be to consider $\operatorname{FTLE}(t, \pm T, x)$ (i.e., use the reference time to be that which includes all the available data, and thus keep $\tau$ fixed) thereby determining the LCSs in the form $\operatorname{LCS}(t, \pm T)$ for $t \in(-T, T)$. Similarly, using $A(t, \pm T, x)$ for (4) in the averaging methods (rather than a fixed time-window length) retains the ability to think of LCS structures as being time-parametrized.

3. Material evolution: The word Lagrangian in Lagrangian coherent structures indicates that LCS are associated with the Lagrangian evolution of solutions of (1). This does not guarantee, however, that LCSs are Lagrangian material curves/surfaces/sets that evolve as material objects under the flow. Material advection is apparently specifiable via the condition $F_{t}^{\tau}(\operatorname{LCS}(t,))=.\operatorname{LCS}(\tau, \cdot)$, but there is an unappreciated issue here: what needs to be put in for the second argument of LCS, i.e., the reference time? Should the same reference time be inserted in both sides of the expression? A reasonable approach might be to put in $T$ (or $-T)$ on both sides, i.e., $F_{t}^{\tau}(\operatorname{LCS}(t, T))=\operatorname{LCS}(\tau, T)$, which is consistent with the infinite-time situation of $T=\infty$. It should be noted that in the time-windowing approaches - such as standard FTLE implementation - material advection would apparently be specifiable by $F_{t}^{\tau}\left(\operatorname{LCS}\left(t, t+t_{w}\right)\right)=\operatorname{LCS}\left(\tau, \tau+t_{w}\right)$, which is clearly inconsistent with the idea of having identical reference times ${ }^{5}$. The reference-time ambiguity does not impact material advection in some LCS methods such as variational LCSs, LAVD or stable/unstable manifolds from asymptotic time extensions. In these cases curves/surfaces are first constrained to flow with (1) (which is a definitive statement), and then subsets of these which satisfy certain conditions are extracted. Other methods are not necessarily guaranteed to obey material advection, because in these reversed approaches LCSs must be identified based on a finite-time flow, and it is only after this that material advection

\footnotetext{
${ }^{5}$ Nevertheless, maximizing ridges of FTLE-fields are seen to approximately obey material advection under some conditions [75]; the reference-time issue is one reason they fail to do so in general [9].
} 
can be examined. Basically, this issue is related to whether $\mathcal{P}$ and $F$ commute.

4. Objectivity/Frame-independence: A view that has been put forward [9] from the continuum mechanics perspective is that any LCS identification method should be frame-independent or objective. This means that if applying an affine transformation of coordinates $y=A(t) x+b(t)$ for any orthogonal matrix $A$ and translation vector $b$ (both potentially time-dependent, and subject to other mild conditions), the LCSs obtained in the $y$-frame should be the same as those in the $x$-frame, suitably transformed. Not all LCS methods satisfy this criterion; those that do include variational LCSs, stable and unstable manifolds from asymptotic time-extensions, transfer operator methods, FTLEs, and the encounter volume method.

5. Implied time-periodicity: As developed, LCS methods analyze structures associated with $F_{t}^{\tau}$, which is a one-step flow map on $\Omega$ from time $t$ to $\tau$. Care should be taken to not impute information beyond the interval $[t, \tau]$ (or $[\tau, t]$ if $\tau<t$ ). Subconscious time-periodic extensions of this interval to $\mathbb{R}$ may be present if applying turnstile dynamics, KAM tori and chaotic transport (these are all associated with repetitively applying a map, such as a time-periodic Poincaré map), using eigenvalues related to $F_{t}^{\tau}$ (an eigenproblem requires mapping from a space to itself; the implicit understanding here would then be that the space at time $\tau$ is the same as that at $t$, i.e., implied time-periodicity), or applying Fourier series in time. Unless there is a specific understanding that time-periodic flows are being considered (which is certainly appropriate in some instances), it should be borne in mind LCSs are transient and only apply in relation to the time interval over which they were computed.

6. Choices of tolerances. In applying the procedure $\mathcal{P}$ to an obtained flow field $F_{t}^{\tau}(x)$, almost all LCS methods will require choosing some parameter values which specify tolerances. For example, if trying to identify an FTLE ridge from an FTLE field, how sharp should the ridge be to qualify? And how is the sharpness defined? For it to be identified as a ridge, how long should it be? In transfer operator methods, how many singular vectors are enough to capture relevant behavior? If using a spectral gap of the singular values for this determination, how should 
one choose that? For clustering methods in general, what are the parameters used for determining whether a trajectory is within or outside a cluster? How small a flux across a curve/surface is allowable for us to identify it as essentially a material curve/surface? What is the appropriate radius to use in the encounter volume approach? Why choose the outermost convex contour as the criterion for low-filamentation in LAVD approaches? And so on. These tolerances/conditions are often chosen such that whatever one seeks to highlight emerges clearly in generated figures and moreover, these chosen tolerances are indistinctly (if at all) specified. We wish to point out that the presence of such tolerances means that extracting LCSs is not as precise a science as is imputed in some studies.

7. Relationships between different LCS methods. Each LCS method uses its own definition for the entities that is seeks. Each remains true to its specific purpose and yields sets that obey that particular objective. Any conclusions about other properties that are not prescribed by the LCS definition are therefore fraught. For example, LCSs defined as maximizing forward-time FTLE ridges are sets with locally the largest stretching rates. However, it is not true in general they are the most repelling material curves, or that they always reveal stable manifolds $[81,82]$; conditions under which these relationships can be anticipated can be found in [82]. Therefore, automatic conclusions on relationships between LCSs obtained from different procedures should be avoided. It is fortunate that there is an increasing interest in determining relationships between various LCS diagnostics [116, 81, 24, 117, 86, 66, 82], to help understand exactly what can be concluded from a particular computation.

\section{Generalized Lagrangian Coherent Structures (GLCSs)}

\subsection{Framework for GLCSs}

We now come to our primary purpose - the introduction of Generalized Lagrangian Coherent Structures, or GLCSs, which capture coherence of variables that co-evolve with the fluid velocity field and whose evolution is governed by an augmented dynamical system.

Let $c$ be the co-evolving variable, defined either as a scalar or vector field on $\Omega$ (generalization to higher-ranked tensors is also possible but we limit 
our presentation to scalars and vectors for simplicity), and which varies with time. Its evolution depends on, but is not solely governed by, the velocity field in (1). Examples of a scalar $c$ include the concentration of a pollutant, the density of a biological organism, or chemical or physical properties of water such as the temperature, salinity, kinetic energy, enstrophy, or components of the vorticity or potential vorticity. When considering the mixing between two fluids, taking $c$ to be the concentration of one of these fluids would be relevant for determining how well-mixed the solution is at each time. Examples of vector $c$ include vorticity in three dimensions, gradients of scalar quantities (e.g., temperature or potential vorticity gradient), or vectors composed of scalar variables such as any of those above. Our goal is to examine these coevolving variables for coherence, rather than confining our attention to fluid trajectories from (1). The evolution of $c$ would be associated with dynamic, chemical, thermodynamic, biological or other relevant governing laws, which are usually coupled to the fluid velocity $v$. Solving for the evolution of $c$ in time requires information about its initial values, $c_{0}(x)$. This is natural, for example, if examining the impact of an oil or other pollutant spill in the ocean. $c_{0}(x)$ need not be smooth in $\Omega$ and could consist of patches or point sources that may be represented using a Dirac delta function. Thus, it is sufficient for $c$ to be a generalized function, or a Schwartzian distribution, i.e., a 'function-like' object whose integral over the domain exists and is finite. To accommodate both scalar or vector $c$ and $c_{0}$, we allow them to take values in $\mathbb{R}^{m}$. More formally, we define:

Definition 2. Let $c_{0}: \Omega \rightarrow \mathbb{R}^{m}$ be a Schwartzian distribution on $\Omega$, denoted $c_{0} \in \mathrm{S}(\Omega)$. This means that for any function $\phi: \Omega \rightarrow \mathbb{R}^{m}$ such that $\phi \in$ $\mathrm{C}^{\infty}(\Omega)$ with compact support, the integral

$$
\left\langle\phi, c_{0}\right\rangle:=\int_{\Omega} c_{0} \cdot \phi \mathrm{d} x
$$

is well-defined and finite.

Just as for LCSs, we seek a definition which identifies subsets of $\Omega$ at a time $t$, with a reference time $\tau$ also being pertinent. Both these times lie in the finite-time interval $[-T, T]$. We will think of $\tau$ as being the 'initial' time at which the scalar field is $c_{0}$, though in the general setting that we describe there is no actual necessity for $\tau$ to be less than $t$. The only requirement is that the $c$ must evolve in a well-posed fashion from a field $c_{0}$ on $\Omega$ at $\tau$, to a field $c$ on $\Omega$ at $t$. 
Definition 3. Let $c(. ; t, \tau): \Omega \rightarrow \mathbb{R}^{m}$ be a co-evolving field at time $t$, which has evolved from a field $c_{0}$ at time $\tau$, and whose evolution is coupled to the flow velocity $v$ through an evolution operator $\mathcal{E}_{\tau}^{t}: \mathrm{S}(\Omega) \times \mathrm{C}^{1}(\Omega \times(-T, T)) \rightarrow$ $\mathrm{S}(\Omega)$ represented abstractly by ${ }^{6}$

$$
\mathcal{E}_{\tau}^{t}\left(c_{0}, v\right)=c(\cdot ; t, \tau) .
$$

More informally, $c(x ; t, \tau)$ is defined for $x \in \Omega$, having taken as inputs functions $c_{0}(x)$ and $v(x, \xi)$ for $\xi \in(-T, T)$.

The equations governing how $\mathcal{E}$ depends on the relevant physics/chemistry/ .../biology and are left unspecified. The easiest example is a tracer $c$ that is being advected by $v$ and diffused, so $\mathcal{E}$ is the advection-diffusion operator. In general, though, it should be noted that $\mathcal{E}$ depends on the initial condition of $c$ at a time $\tau$, and the velocity field in (1) during the entire time-domain of interest. Both of these, as well as the particular evolution operation $\mathcal{E}$, are used to compute $c(x ; t, \tau)$ on $\Omega$. Having obtained the co-evolving field $c(x ; t, \tau)$, the next issue is the extraction of relevant coherent entities of $c$ at the time-instance $t$. We term the sets of $\Omega$ identified from this process Generalized Lagrangian Coherent Structures (GLCSs), and we assume that these are found by performing an operation on the co-evolved vector field according to

$$
\operatorname{GLCS}(t, \tau):=\mathcal{P}(c(\Omega ; t, \tau)) .
$$

Following the notation as for LCSs, we use the first argument of GLCS to identify the time at which the GLCS sets are identified, and the second argument the reference time, which in this case is $\tau$ (i.e., fluid velocities for times between $t$ and $\tau$ are utilized in the computation). Just as for LCSs, the particular procedure $\mathcal{P}$ shall be left unspecified. This generalized methodology allows us to extend the notion of finite-time coherence to any co-evolving fields $c$ described by additional physics, chemistry, thermodynamics, or biology, and also subject to initial conditions $c_{0}$, instead of confining ourselves to trajectories of (1).

We now show why LCSs are a special case of the GLCS framework. Let $c_{0}$ be the position/identity function on $\Omega$, i.e., $c_{0}\left(x_{0}\right)=x_{0}$ represents the

\footnotetext{
${ }^{6}$ We have avoided writing $(10)$ as, for example, $\mathcal{E}_{\tau}^{t}\left(c_{0}(x), v\right)=c(x ; t, \tau)$ because this would mistakenly indicate that the same $x$ value was being inserted in both sides. The notation $c(. ; t, \tau)$ indicates a function over the first argument (i.e., a vector field on $\Omega$ ), with the second and third kept fixed at $t$ and $\tau$.
} 
co-evolution field at time $\tau$. We want to examine GLCS at the time $t$. We define the co-evolution field for $x \in \Omega$ at time $t$ by

$$
c(x ; t, \tau):=c_{0}\left(F_{t}^{\tau}(x)\right)=F_{t}^{\tau}(x) .
$$

where the second equality occurs because of the choice of $c_{0}$ as being the identity. The first equality (the definition) states that the value of the coevolution field at a location $x$ at time $t$ is obtainable by evaluating $c_{0}$ at the location to which $x$ flows to by time $\tau$, where the flow is according to the ordinary differential equation (1). This choice of co-evolution is therefore linked to particle positions, and in this case (11) reduces to

$$
\operatorname{GLCS}(t, \tau)=\mathcal{P}(c(\Omega ; t, \tau))=\mathcal{P}\left(F_{t}^{\tau}(\Omega)\right)=\operatorname{LCS}(t, \tau) .
$$

Thus, the GLCSs at the time instance $t$ (with reference time $\tau$ ) are equivalent to LCSs with the same understanding of time. GLCSs are therefore a generalization of LCSs.

Another way to think of this relationship is that in LCS methods, one establishes a connection between the vector field $x$ and the vector field $F_{t}^{\tau}(x)$, which are relevant at the time-instances $t$ and $\tau$ respectively. In GLCS methods, the relationship is between the vector fields $c(x ; t, \tau)$ and $c_{0}(x)$, which are associated with the time-instances $t$ and $\tau$ respectively. In other words, the LCS vector fields are very specific (particularly the identity/position vector field associated with time $t$ ), and can be represented as an invertible map between the two times because ordinary differential equations are reversible in time. In contrast, the vector fields for GLCSs are more general, and moreover the relationship between them may not be invertible (e.g., diffusion equations are only well-posed in the forward time direction).

\subsection{Quantities for which GLCSs may be necessary}

Of course, our goal is not just to provide a definition that reduces appropriately to that of standard LCSs; more interesting is the way that our definition can be used to think about coherence for more general, augmented dynamical systems. In this light, we now give some examples of physically relevant choices for $c$. In the following sections, we will discuss some of these examples in more detail, showing explicitly how standard LCSs can fail to capture their dynamics, but also limits for which standard LCSs will appropriately diagnose them. 
(a) Suppose that $c$ is the concentration field of some passively advected tracer. Then, in our notation, $c(x ; t, \tau)$ gives a function on $\Omega$ which expresses the concentration distribution at $t$, subject to some initial concentration $c_{0}(x)$ at a reference/initial time $\tau$. In a given problem of interest, such a scalar $c$ could represent a pollutant concentration or some other kind of transported observable like the sea-surface temperature [56, 44, 45] or color [84]. A simplest model for the evolution of such scalar fields could be an advection-diffusion equation of the form

$$
\frac{\partial c}{\partial t}+\nabla \cdot(c v)=\frac{1}{\mathrm{Pe}} \nabla^{2} c+s(x, t)
$$

where Pe is the Péclet number (proportional to the reciprocal of the diffusivity) and $s$ is some source/sink term. That is, the quantity $c$ of interest to us is a scalar density that evolves by getting carried along by the flow of (1) while also diffusing [118, 119] and potentially changing its total amount (via $s$, e.g., ocean pollutants sinking to the bottom). An alternative statement of this passively advected-diffused situation is that $\mathcal{E}$ in this instance is the transfer operator as discussed in Section 2 in the limit $\mathrm{Pe} \rightarrow \infty^{7}$. The procedure $\mathcal{P}$ would entail extracting relevant features (e.g., uniform areas, sharp gradients) from the field $c(x ; t, \tau)$.

Depending on exactly what physical quantity $c$ represents, the GLCSs associated with its evolution will convey different physical insights. If $c$ were the oceanic temperature, the evolution of the GLCSs with time could help track the movement of oceanic hotspots (or coldspots). Their importance is that they affect the heat transport in the ocean, and through coupling with the atmosphere, also impact the weather system. Similarly, if $c$ were the sea-surface color, which is correlated with the plankton density [84], then the GLCSs would be associated with biological activity in the ocean.

In the limits of $\mathrm{Pe}=\infty$ and $s \equiv 0$, and if additionally $\nabla \cdot v=0$ (i.e.,

\footnotetext{
${ }^{7}$ There are subtleties associated with this limit (diffusion going to zero) when comparing (1) with the advective-diffusive flow (13) with $s \equiv 0[120,88]$; these are beyond the scope of the present paper. However, it should be noted that when computationally determining how densities are transported by (1) as discussed in Section 2, 'diffusion' occurs implicitly through the numerical discretization process.
} 
the fluid is incompressible), (13) becomes

$$
\frac{D c}{D t}:=\frac{\partial c}{\partial t}+v \cdot \nabla c=0,
$$

a statement that $c$ is conserved along trajectories of (1). If so, understanding LCSs will often give insights into the GLCSs associated with $c$. However, depending on the initial distribution of $c$, the GLCSs that one may observe might be different from the LCSs, since LCSs do not take this initial distribution into account. We return to this example in detail in Section 4.

Depending on the situation, slightly different forms from (13) may be in order: non-constant diffusivity, reaction terms governing chemical processes, coupling with other evolution equations (e.g., Boussinesq approximation for the temperature), etc.

It must also be mentioned that (13) or closely related equations need to be considered in recent methods such as 'dynamic Laplacian' [120, 121] or 'geometric heat flow' [122]; these augment purely advective motion (i.e., LCS in our notation) with an explicit characterization of diffusion. Thus these emerging methods also fall under our GLCS framework.

(b) In some cases, particles/densities move according not just to the fluid velocity, but are also influenced by additional velocities. A simple example of this is of a jellyfish swimming, thereby modifying the adjacent fluid velocity [114]. An ad hoc way of determining the impact of this on the density of a quantity (e.g., nutrient, pollutant, plankton) is to simply compute the LCSs associated with the modified velocity. In this case, one sets $c$ not from the flow map of the fluid velocity as in (12), but from a flow map $\tilde{F}$ associated with the fluid velocity plus its modification. Applying an LCS method (e.g., FTLEs as in [114] for jellyfish swimming) and extracting ridges would give flow structures that are a skeleton for how the density would evolve. Unlike the advectiondiffusion approaches, this does not explicitly incorporate diffusion, and moreover does not deal with a particular density distribution.

(c) If $c=\nabla \times v$, then $c$ is the vorticity, which is advected by the velocity, diffused by the kinematic viscosity, tilted and stretched (in three dimensions) by its coupling to the strain field, and potentially modified by nonconservative forcing. Since $c$ depends on the velocity $v$ in this 
case and can feed back on it, $c$ is now a dynamically active scalar. In this situation, $v$ and $c$ evolve with mutual interdependence, as given by the vorticity equation. Other active scalars, such as the kinetic energy or rate of viscous energy dissipation, also have a dependence on $v$. A GLCS approach that incorporates the evolution equation is necessary to determine structures associated with $c$ in these instances.

(d) Suppose we have a mixture of $m$ immiscible different fluids, such as in a co-flow or an emulsion, and we think of evolving blobs of each of these fluids as our GLCS. We can tag each of these fluids with a numerical label, using 1 for fluid 1, 2 for fluid 2, and so on up to $m$ for fluid $m$. One way to define a relevant field $c(x ; t, \tau)$ is to represent it as an $m$ dimensional vector, whose $j$ th component is an indicator function for fluid $j$ (there are other possibilities as well). We will address a example of this form (with only two fluids) in Section 4.2.

(e) A situation conceptually intermediate between an advected passive scalar and a field containing multiple fluids is that of front propagation. In this case, the scalar field (e.g., temperature, chemical concentration) is characterized by a sharp boundary between high and low (or vanishing) value. When these fronts propagate within a fluid, the local front speed depends not just on the fluid speed but also on the front's orientation. If $x=\left(x^{(1)}, x^{(2)}\right)$ is a point that lies on a front within a two-dimensional flow in which the fluid velocity is $v(x, t)=\left(v^{(1)}, v^{(2)}\right)$, a standard way of modeling the front's evolution is the system $[32,33,34,35,36]$

$$
\begin{gathered}
\dot{x}^{(1)}=v^{(1)}+\alpha \sin \theta \quad, \quad \dot{x}^{(2)}=v^{(2)}-\alpha \sin \theta, \\
\dot{\theta}=-2 v_{x_{1}}^{(1)} \cos \theta \sin \theta-v_{x_{2}}^{(1)} \sin ^{2} \theta+v_{x_{1}}^{(2)} \cos ^{2} \theta .
\end{gathered}
$$

Here, $\theta$ represents the local orientation between the front propagation velocity (defined to be normal to the front, at speed $\alpha$ ) and the fluid velocity $v$, and the subscripts indicate partial differentiation. It has been established [123] that the stable and unstable manifolds associated with the system (15) restrict how fronts propagate, and thus the GLCSs are associated with applying an operation $\mathcal{P}$ to the flow map of (15). This is in contrast to applying $\mathcal{P}$ to fluid particle propagation alone, which is given by (1) and which would give LCSs associated with particle positions. This is another example in which the GLCSs 
can be thought of as applying LCS methods to an augmented velocity field (in this case, in one higher dimension). Similar ideas may potentially be used for fronts of active scalars such as the momentum at a turbulent/non-turbulent interface.

(f) LCSs identify structures associated with the advection of fluid parcels. But if we instead consider noninfinitesimal solid particles advected by the flow - typically known as inertial particles, since they behave as if they have inertia relative to the carrier fluid-the situation changes. The trajectories of inertial particles are not simply the solutions of (1). Rather, they follow the Maxey-Riley equation [124], and feel a host of effects, such as added mass, drag, lift, and history forces, in addition to simple advection. Thus, GLCSs are again required. There has been some work attempting to extend simple LCSs to capture their behavior $[111,112,113]$, but more work remains to be done. A particular complicating factor for inertial particle dynamics is the fact that the Basset history force makes their evolution nonlocal in time, in that the force on an inertial particle at an instant depends on its entire previous history. A "velocity field" constructed from an ensemble of inertial particles thus need not be single-valued or differentiable, and the relative velocity of two inertial particles at contact does not have to vanish $[125,126]$.

(g) In attempting to evaluate the heat flux which is coupled to a fluid velocity field, Kimura and Bejan [127] proposed the concept of 'heatlines' which serve as effective streamfunction contours for the flow of heat in a two-dimensional incompressible steady flow. Building on this, other authors have suggested forming an effective velocity field which captures the impact of the fluid velocity on the flux of a relevant scalar, including also the possibility of diffusion [127, 128, 129, 130, 131, 132]. Thereafter, a standard LCS method could be applied to this velocity field $[132,133]$. These approaches fall exactly within our framework of GLCSs, with the co-evolution being associated with the advection by the effective velocity field. We will demonstrate the usage of one of these methods [133] in an example related to the evolution of seasurface temperature in Section 4.3.1.

(h) There is an emerging need for understanding the impact of stochasticity in velocity fields in, for example, attempting to quantify the impact of 
unresolved small scales on large scale motions ${ }^{8}$ [134]. In the oceans and atmosphere, there is evidence of such stochasticity which, when computed in terms of an effective diffusivity from data, is both spatially and temporally heterogeneous $[135,136,137,138]$. The approach in $[48,139,140,141]$ is to have the evolution operator be the flow of the stochastic differential equation

$$
\mathrm{d} x_{t}=v\left(x_{t}, t\right) \mathrm{d} t+\varepsilon \sigma\left(x_{t}, t\right) \mathrm{d} W_{t}
$$

in which $|\varepsilon|$ is small, $\sigma$ is an $n \times n$ diffusion matrix, and $W_{t}$ is Brownian noise. Extracting sets from $\Omega$ based on statistical fields (such as mean and variance) arising from the evolution from time $\tau$ to $t$ would then constitute the procedure $\mathcal{P}$ for GLCS determination [139, 140, 141].

Some of the GLCSs mentioned above fall within the framework of a transport equation

$$
\frac{\partial c}{\partial t}+\nabla \cdot \Phi=s
$$

in which $\Phi$ is a $v$-dependent flux, and $s$ is a source term. For example, the advection-diffusion equation (13) is obtained by choosing $\Phi=c v-\nabla c / \mathrm{Pe}$, and (14) emerges if $\Phi=c v$ and the fluid is incompressible. Similarly, the heatline approach $[127,132]$ uses $\Phi=\alpha c v-\beta \nabla c$ in which $c$ is now the temperature, and $\alpha$ and $\beta$ are constants associated with specific heat and thermal conductivity. Situations in which a modified velocity field $v^{\star}$ rather than $v$ is used ${ }^{9}$ are obtained by setting $\Phi=c v^{\star}$ and $s=0$. The standard LCS framework is related to (17) by choosing $\Phi=c v$ and $s=0$, and considering this with a uniform initial distribution $c_{0}$. (However, the basic implementation of the LCS method is easier done using (1) directly, as opposed to the more abstract representation (17) under these conditions.)

\section{Examples comparing GLCSs to LCSs}

In this section, we consider three examples in detail. In each case, we will compare some LCS calculations with a particular GLCS calculation.

\footnotetext{
${ }^{8}$ This is sometimes referred to as the 'stochastic parametrization' problem, and is seeing intensive investigation in global climate and ocean flow models.

${ }^{9}$ While the burning manifold approaches (15) are indeed associated with such a modified velocity field, fitting it into the framework (17) in this case has the discrepancy of being in a higher dimension.
} 


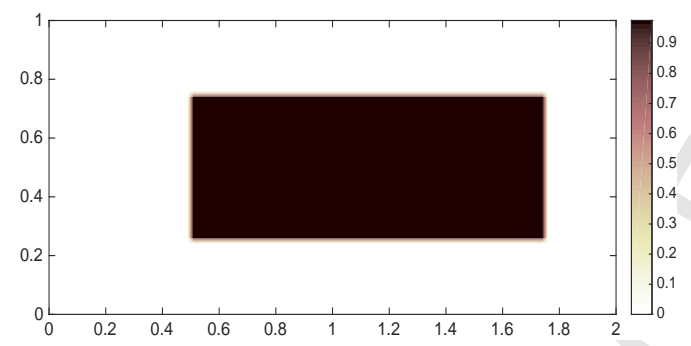

Figure 1: The initial $(\tau=0)$ pollutant concentration, which is the primary one used in the discussion of Section 4.1. The intention is to discuss structures in the evolved concentration field at a later time $t=1$.

\subsection{Passive scalar advection}

The term "passive scalar field" is typically used to describe some scalar quantity that is transported by the fluid flow but that does not couple back to the flow and affect it. Thus, generically, the dynamics of a passive scalar will be the solution to some advection-diffusion equation, since the scalar may have some diffusivity relative to that of the fluid (that is, a Schmidt number different from unity). The most common example of a passive scalar is the concentration of a contaminant, often a dye, chemical, or pollutant; in some cases, however, temperature, salinity, or other scalar quantities may also be thought of as passive.

We consider the domain $\Omega=[0,2] \times[0,1]$, and the initial $(\tau=0)$ pollutant concentration being that of a 'patch' as shown in Fig. 1. The GLCS we seek are associated with how this has spread by time $t=1$. The spreading will be modeled by equation (13), but we additionally assume that the fluid is incompressible, and there are no sources or sinks. Then, (13) becomes

$$
\frac{\partial c}{\partial t}+v \cdot(\nabla c)=\frac{1}{\mathrm{Pe}} \nabla^{2} c .
$$

We specify an unsteady velocity field $v$ that has seen extensive use as a testbed for LCS analysis [142, 143, 109, 144, 1, 145, 38, 146, 102, 77, 147, 78, $79,148,76,88]$ : the double gyre flow as introduced by Shadden et al [75]. The velocity $v=\left(v_{1}, v_{2}\right)$ takes the form

$$
\left.\begin{array}{l}
v_{1}\left(x_{1}, x_{2}, t\right)=-\pi A \sin \left[\pi h\left(x_{1}, t\right)\right] \cos \left[\pi x_{2}\right] \\
v_{2}\left(x_{1}, x_{2}, t\right)=\pi A \cos \left[\pi h\left(x_{1}, t\right)\right] \sin \left[\pi x_{2}\right] \frac{\partial h}{\partial x_{1}}\left(x_{1}, t\right)
\end{array}\right\},
$$




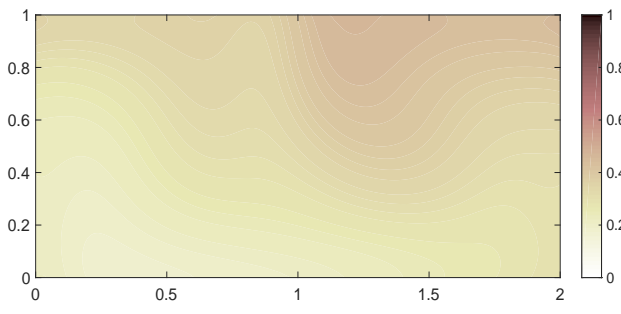

(a) $\mathrm{Pe}=10$

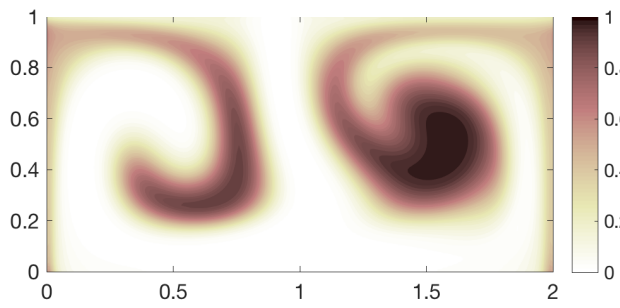

(c) $\mathrm{Pe}=300$

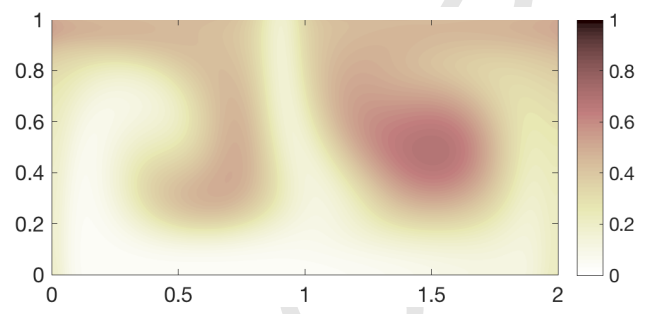

(b) $\mathrm{Pe}=50$

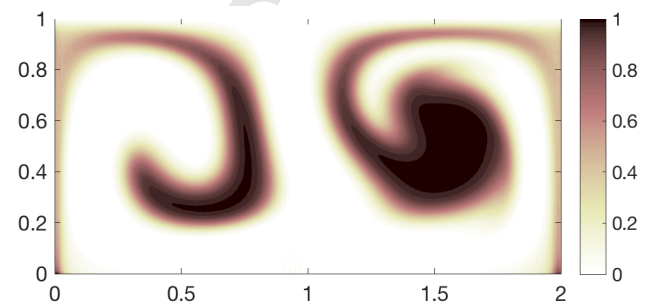

(d) $\mathrm{Pe}=800$

Figure 2: Evolution of the 'patch' pollutant distribution of Fig. 1 from $\tau=0$ to time $t=1$ using (13) and the double gyre velocity field (19), using several different values for Pe.

where the function $h$ is defined by

$$
h\left(x_{1}, t\right):=\varepsilon \sin (\omega t) x_{1}^{2}+[1-2 \varepsilon \sin (\omega t)] x_{1} .
$$

It is well-known that for sufficiently small $|\varepsilon|$, the double-gyre flow possesses hyperbolic trajectories near $(1,0)$ and $(1,1)$, which move with time. The stable manifold of the former trajectory and the unstable manifold of the latter extend around $x_{1}=1$, intermingling to generate provably [142] chaotic motion, separating a clockwise-rotating gyre on the left from a anticlockwiserotating gyre on the right.

In all the calculations presented, we use the parameter values $A=1$, $\varepsilon=0.3$ and $\omega=10 \pi$, and the spatial resolution $0.01 \times 0.01$. We evolve $(18)$ using a pseudo-spectral code, with time-stepping performed by a CrankNicholson routine, and show the results for several choices of Pe at $t=1$ in Fig. 2. We will use this brown color scheme consistently for concentration fields (for usage in identifying GLCSs) in what follows, to distinguish from the green/yellow scheme used for scalar fields associated with identifying LCSs. From Fig. 2, we observe that when Pe is small, the pollutant smears out rapidly (thereby not having any obvious patterns), whereas when $\mathrm{Pe}$ is large, discernible patterns emerge. There is a faint ridge of the pollutant 


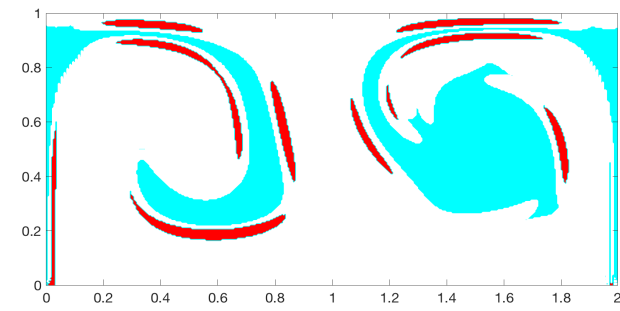

(a) $\alpha=0.3, \beta=0.1, \gamma=0.3$

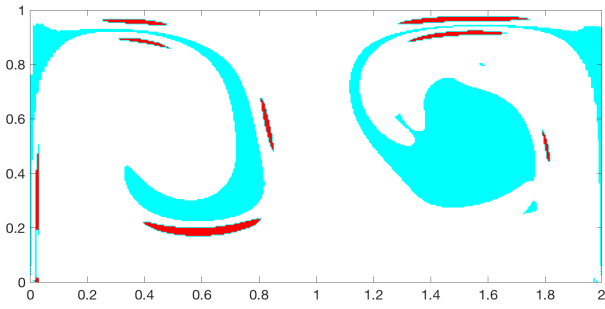

(c) $\alpha=0.3, \beta=0.05, \gamma=0.4$

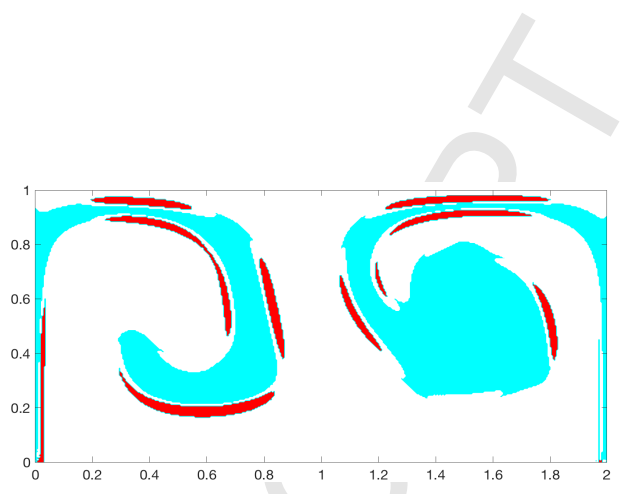

(b) $\alpha=0.4, \beta=0.2, \gamma=0.3$

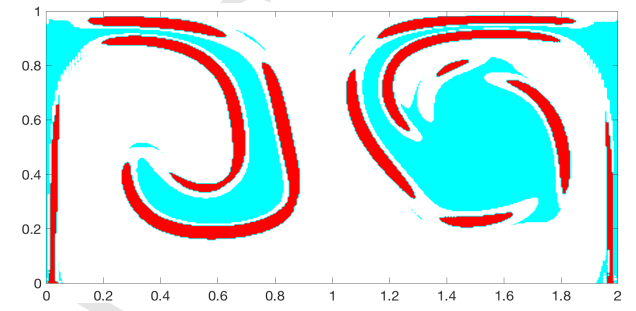

(d) $\alpha=0.2, \beta=0.1, \gamma=0.2$

Figure 3: Extraction of GLCSs as sharp transitions (red) and uniformly-mixed high density regions (cyan) from the $\mathrm{Pe}=800$ situation (Fig. 2(d)), using different threshold values as described in the text.

concentration nearby $x_{1}=1$ when $\mathrm{Pe}=300$, and for large Pe, pollutant appears to be more dominantly entrained into twirling structures on the two sides. It should be emphasized that the structure of the pollutant concentration at time $t=1$ must depend on both the Péclet number ${ }^{10}$ and the initial pollutant concentration.

Now, we might be interested in sharp boundaries between disparate density regions, and uniformly mixed regions of high density, as our 'important structures.' Consequently, the GLCSs in this situation might be respectively construed to be filaments associated with anomalously large values of the density gradient, and regions where the density is mostly uniform but the density is fairly high. Thus, the 'coherence' in the pollutant concentration is associated with the magnitude of the gradient of the concentration; large values would indicate sharp transitions and small values would indicate uniform regions. Extracting such regions immediately leads to the standard ambiguity of having to define threshold values. In Fig. 3, we use different

\footnotetext{
${ }^{10}$ Equivalent to making Pe large is making $t$ small, since Pe is related to the diffusive timescale, though this can have different spatial influences depending on the local densities.
} 
threshold values to extract these two types of GLCSs from Fig 2(d): red to indicate sharp boundaries, and cyan for well-mixed large density regions. We let $\alpha$ be the fraction of the maximum density which is considered 'high,' $\beta$ the fraction of the maximum density gradient below which we consider the flow well-mixed, and $\gamma$ the fraction of the maximum density gradient above which we define the density as undergoing a sharp transition. The red (sharp transition) regions are defined as having density gradient larger than $\gamma$ times the maximum density gradient, and the cyan (uniform density) regions have density gradient less than $\beta$ times the maximum density gradient, but have a density greater than $\alpha$ times the maximum density. Identifying the GLCSs from Fig. 2(d) according to this prescription with several different choices of these threshold values is shown in Fig. 3. Clearly, depending on the threshold values (i.e., the procedure for extracting the GLCSs), different sets will be identified from the diagnostic GLCS field of Fig. 2(d). Therefore, when discussing connections to LCSs, it will be opportune to compare with the density fields in Fig. 2 - which possess more information than in the extracted GLCS pictures of Fig. 3. Moreover, we remark that for the smaller Pe situations pictured in Fig. 2(a-c), identification of sharp boundaries and uniform densities becomes more ambiguous as Pe decreases.

For comparison with Fig. 2, in Figs. 4 and 5 we present several approaches to finding $\operatorname{LCS}(1,0)$, i.e., LCS structures at time $t=1$, which takes into account the flow from time $\tau=0$. The left panels in Fig. 4 are the computed diagnostic fields obtained from the flow from time 1 to 0 , and the right panel shows extracted LCSs based on some simple choices of procedures $\mathcal{P}$. We do not use the most sophisticated methods available for such extraction, because (i) we are only interested in a qualitative comparison, and (ii) more refined extraction methods will result in approximately the same entities. We moreover emphasize that our intention is not to compare or critically evaluate different LCS methods (readers are referred to papers such as $[110,143]$ for this), but rather to illustrate that results arising from different LCS methods are not necessarily the same, and do not necessarily have a connection to GLCS sets chosen according to some specification.

The top row is the backward FTLE field computed using (8). We extract the LCS as the 'strongest ridge' which in this case is obtained by simply thresholding by $80 \%$ of the maximum value of the FTLE field ${ }^{11}$. The next row

${ }^{11}$ There are more sophisticated ways of extracting ridges $[149,150]$, but here we use the 

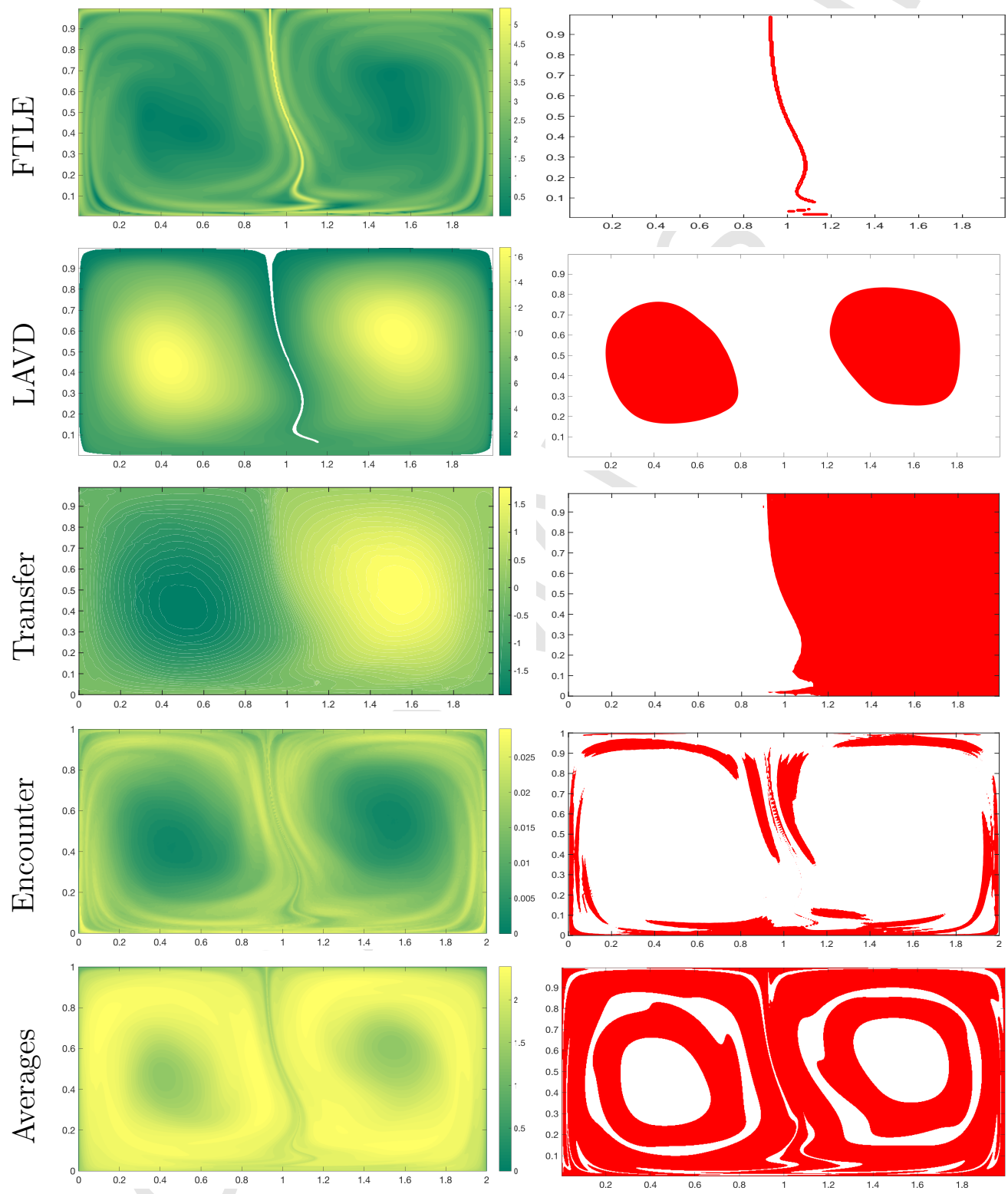

Figure 4: Obtaining $\operatorname{LCS}(1,0)$ for the double-gyre flow using several methods. The left panels show the relevant diagnostic fields, and the right shows extracted LCSs in red. Detailed descriptions are available in the main text. 
is the LAVD field computed using (5) and (4). The white areas in the LAVD field indicate that the numerical computation fails for these regions, because particles seeded in these areas approach the boundaries during the backwardtime advection, at which the vorticity becomes undefined because the velocity field is undefined on the outside of the boundary. The fact that the undefined white region in the center of the LAVD picture matches with the FTLE ridge is no coincidence; this ridge corresponds to the unstable manifold emanating from a point on the boundary. In the LAVD case, the suggestion is that the region within 'the outermost closed convex contour' [63] provides the LCS. Using a trial-and-error investigation of contours, we thus obtain the red set in the right-hand figure. The FTLE- and LAVD-generated LCSs in the right figures illustrate the two principal types of LCSs respectively: those associated with strong stretching, and those with strong coherent rotation. The former can be thought of as a barrier between coherent entities (with eventual behavior being highly sensitive to mild perturbations nearby), while the latter comprises coherent vortex-like structures which rotate robustly with little filamentation developing.

The next row is obtained using the transfer operator approach $[89,90,88]$. The left figure is the singular vector associated with the second-top singular value of the transfer operator, i.e., the probability transition matrix from one pixel to another. The right figure has been obtained by simply partitioning at the zero contour, resulting in two sets (white and red) which are 'almost coherent' [90] under the flow from time 1 to 0. It is possible (not shown) to obtain higher-order 'almost coherent' sets by examining the third, fourth, etc, singular vectors; these can help refine the vortex areas. The next row of LCSs is computed using the encounter volume approach [100, 101], which provides a measure of how fluid parcels encounter each other over the course of the flow. High values indicate that fluid parcels in this region encounter many other fluid parcels during the duration, and the right figure is obtained by partitioning the left at a threshold of $70 \%$ of the maximum encounter volume to obtain regions with high mixing ${ }^{12}$. It should be noted that all methods discussed so far respect objectivity (invariance under time-dependent frame

simplest methods for easy description and comparison.

${ }^{12}$ Because encounters are caused by both strain and shear, the highest encounter volumes are not necessarily along the stable manifold. For longer integration times (if we integrate back to $\tau=-3$, for example), strain along the manifold wins over the shear and the encounter volume field focusses more on the manifold. 


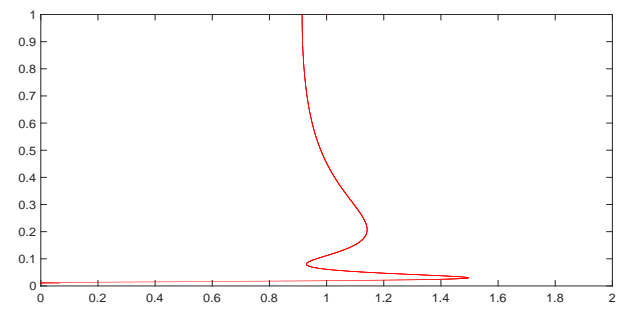

(a) Unstable manifold (approximation)

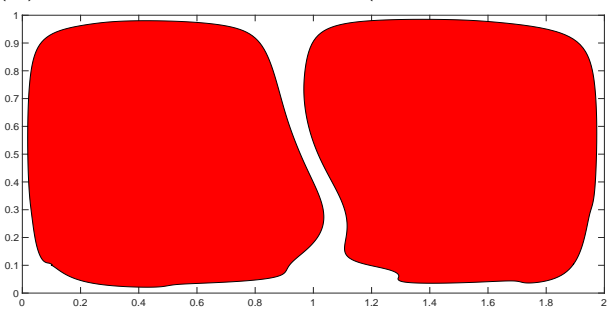

(c) Variational elliptic LCS

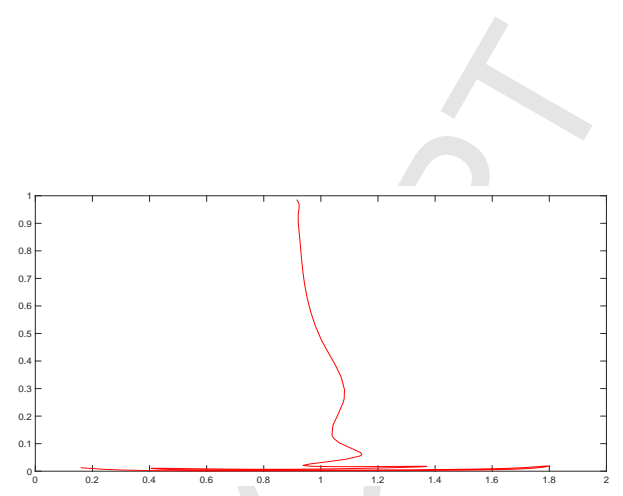

(b) Unstable manifold (numerical)

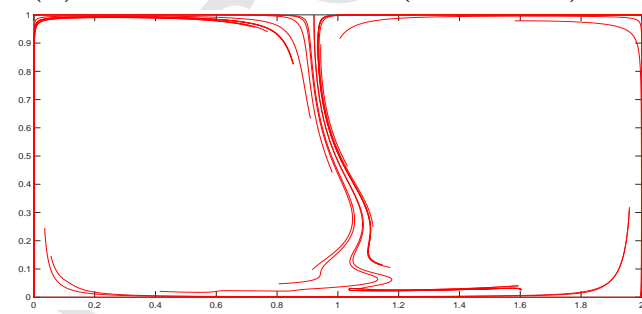

(d) Variational hyperbolic LCS

Figure 5: Finding $\operatorname{LCS}(0,1)$ for the double-gyre using several methods which do not per se rely on extraction from a scalar field.

transformations).

The final panel plots on the left the Lagrangian average of the speedi.e., (4) with $g=|\cdot|$ and $b=v(\cdot)$-as proposed by $[151,60]$. This particular technique does not come with a specified way of extracting structures from the field, and so on the right we present structures obtained from a slightly different Lagrangian average, the gradient of the mesochronic velocity field $[61,62]$ which is obtained by averaging the velocity as opposed to the speed. Regions in which the eigenvalues of this are real are defined as mesohyperbolic, and it is these regions which are shown in red [61], and extracted here as our LCS. The white regions are mesoelliptic [61], and this classification is meant to partition particles which experienced 'hyperbolicity on average' over the time-interval (these are the red mesohyperbolic regions) from those which did not (mesoelliptic regions). Both the left and right pictures indicate vortex cores (mesoelliptic regions), which are surrounded by high values of the average (mesohyperbolic regions on the right). These pictures offer contradictory insights in comparison to the FTLE and LAVD pictures, illustrating the fact that not all LCS methods are equivalent, or seek the same sorts of things.

A different class of LCS methods in which the LCS structures are directly 
obtained from some procedure $\mathcal{P}$ on the velocity field, without necessarily being extracted from a particular scalar field ${ }^{13}$, are displayed in Fig. 5. The top row contains computations of the unstable manifold at $t=1$, to define which it is necessary to extend the flow backwards beyond $\tau=0$; in this case we imagine that it has been done time-periodically ${ }^{14}$. In (a), we use an explicit approximation ${ }^{15}$ to the unstable manifold using Melnikov theory for small $\varepsilon[1]$. In (b), we obtain the manifold numerically; because this is a nonautonomous situation, we cannot simply seed particles near a fixed point. We therefore use the numerical procedure for computing nonautonomous manifolds described in [152]. The numerically determined unstable manifold has a strong relationship to the FTLE ridge in Fig. 4. The second row in Fig. 5 relates to the variational definition of LCSs due to Haller $[9,81]$, in which curves which experience extremal repulsion (in backward time) are classified. Both computations were performed using LCS Tool [70] for our parameter values, and using the default tolerances in the package. The left figure shows the interior of two elliptic LCSs, curves which are stationary with respect to averaged tangential stretching [9, 153]. The right figure shows the hyperbolic LCSs - in this case curves from which there is maximum repulsion over the backward-time flow $[9,81]$. The intuition is that these variational LCSs are associated with the vortex cores and unstable manifold curves respectively. There are relationships evident with other LCS methods displayed in Fig. 4.

None of the LCS methods are designed to take into account either the initial pollutant distribution or its subsequent evolution with time, and hence there are no compelling connections to the pollutant concentration pictures of Fig. 2. Put another way, the LCS method used simply takes all initial conditions, with equal weight, and advects them according to (1) with no

\footnotetext{
${ }^{13}$ We may alternatively think of these as a scalar field consisting of an indicator function on these sets.

${ }^{14}$ In any extension, it will be necessary to obey the exponential dichotomy $[21,22$, 23] property to ensure that hyperbolic structures to which are attached stable/unstable manifolds are retained. The differences in the manifolds based on different extensions can be quantified locally using techniques such as those discussed in [67]; the impact of these extensions get damped exponentially in time, and so there is usually little visible difference for 'reasonable' extensions.

${ }^{15} \mathrm{An} \mathcal{O}(\varepsilon)$ expression $x_{1}=1-\frac{\varepsilon \pi^{2} A}{2 \sin \pi x_{2}} \int_{-\infty}^{1} \sin \left[4 \cot ^{-1}\left(\cot \frac{\pi x_{2}}{2} \exp \left[\pi^{2} A(\tau-1)\right]\right)\right] \sin \omega \tau \mathrm{d} \tau$ is obtained from (2.70) in [1] (see also [142]); since $\varepsilon=0.3$ here, we do not get a good result.
} 


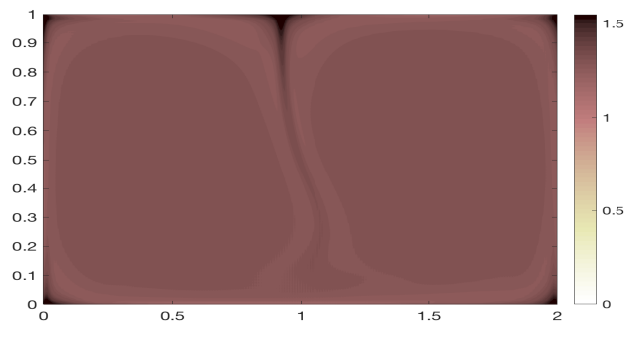

(a)

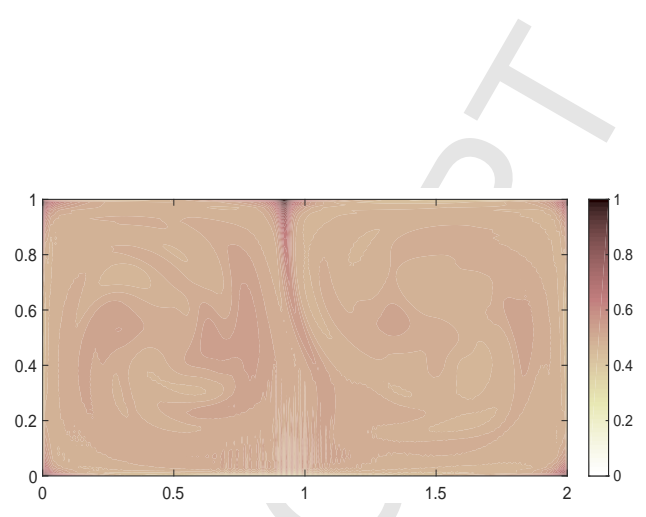

(b)

Figure 6: Evolved densities by $t=1$ using $\mathrm{Pe}=800$, using (a) a uniform, and (b) a random, initial distribution $c_{0}$.

regard to the pollutant's spatial distribution. Furthermore, the LCS does not take into account the laws governing the evolution of the pollutant density. Given this, it is clearly the height of optimism to expect any relationship between what we are primarily interested in (viz., structures associated with how the pollutant spreads) with information obtained from LCSs. On the other hand, if $\mathrm{Pe} \rightarrow \infty$, then we note that (18) collapses to insisting that $c$ remains constant along trajectories. Therefore, trajectory-derived LCS ridges/patches may be similar to ridges/patches of the $c$ field as $\mathrm{Pe} \rightarrow \infty$ if $c_{0}$ is uniform, or if $c_{0}$ is such that relevant regions are appropriately sampled. However, this may be impractical because numerically advecting high Péclet number flows is prone to accuracy/stability problems [154]. We show in Fig. 6 situations in which we use for $c_{0}$ uniform and random ${ }^{16}$ distribution. Both the 'ridge' and the 'vortex core' regions are discernible in the uniform implementation.

We summarize our observations from the computations. For the particular instance of the co-evolving quantity being an advection-diffusion driven tracer field, for there to be a similarity between LCSs and GLCSs, Pe $\rightarrow \infty$ is necessary. Secondly, the initial distribution of tracer $c_{0}$ should be such that it samples the specific regions associated with the particular LCS that it is to be compared to, or otherwise evolves to a stage where it does so. Defining exactly what conditions this imposes on $c_{0}$ is difficult. Stating this difference between GLCSs and LCSs another way, the tracer density evolution depends on the choice of $c_{0}$, whereas there is no such prejudicing of regions in standard

\footnotetext{
${ }^{16}$ We need to refine the grid to $0.005 \times 0.005$ in these cases, and for the random situation allocate each spatial box with an initial density value in $(0,1)$, uniformly sampled.
} 
LCS methods. Thirdly, the time of flow could also be a factor: if too short, high densities may not reach relevant areas, and if too long, diffusion will smear out all density anomalies. Unless all these conditions - sufficiently high Pe and compatible initial condition and time - are satisfied, using LCSs as a predictor of GLCSs in this advection-diffusion situation is essentially useless. In some situations in which the FSLE field imputed from oceanic velocities has similarities with the ocean temperature field $[56,44,45]$, presumably the "correct" conditions are approximately satisfied.

Here, the coupling between $c$ and the velocity field $v$ obeyed (18). If their relationship was given by the more general transport equation (17), then once again certain LCS/GLCS similarities can be imputed in limiting instances, notably in situations when the generalized velocity $v^{\star}$ approaches $v$. Claiming LCS/GLCS relationships is questionable when more general co-evolution laws are in operation.

\subsection{A flow interface}

In this example, we examine the 'coherence' associated with two fluids in a channel, with the evolution of their mutual interface being the GLCS of interest. The prototypical flow we consider is that of a cross-channel micromixer $[155,156,157,158,159,160,161,162]$, with the two fluids flowing parallel to one another from left to right in a main channel, as pictured in Fig. 7. In addition, there are several small cross-channels, in which fluid is made to travel in an unsteady way by attaching to syringes or pumps ${ }^{17}$. This configuration can lead to unsteady velocities transverse to the primary flow within the main channel, thereby attempting to make the flow interface more complicated. The GLCS in this instance will be the time-varying flow interface, which we will obtain numerically. We will consider the cases where the two fluids are immiscible as well as miscible.

We label our coordinates as $x=\left(x_{1}, x_{2}\right)$. Suppose the main channel has width $L$, but that fluid 1 (upper fluid) enters at twice the flow rate of fluid 2 (lower). This means that the flow interface between the fluids, in the absence of cross-channel flow, is located $2 / 3$ of the way down from the top. Thus, let $-L / 3<x_{2}<2 L / 3$ define the $x_{2}$-coordinate of the main channel, for which

\footnotetext{
${ }^{17}$ Transverse velocities are alternatively obtainable by having flexible membranes as the walls of the main channel, which are pulsated in some fashion [163, 164, 162].
} 


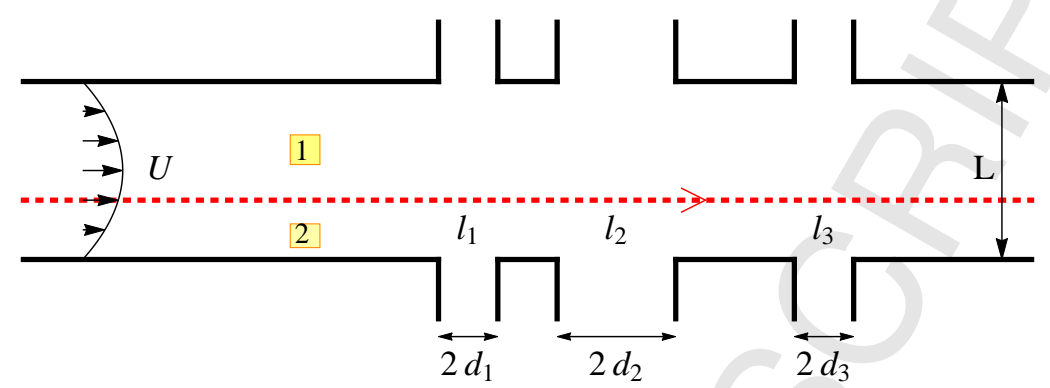

Figure 7: Cross-channel micromixer with three cross-channels; the two different fluids are labeled 1 and 2 , and are separated by an interface along $x_{2}=0$.

a standard parabolic flow profile gives the velocity

$$
v_{1}\left(x_{2}\right)=\frac{4 U}{L^{2}}\left(\frac{2 L}{3}-x_{2}\right)\left(x_{2}+\frac{L}{3}\right)
$$

where $U$ is the maximum speed (at the central location). With this choice of coordinates, $x_{2}=0$ is the flow interface between fluids 1 and 2 . This is therefore the steady GLCS, and the speed along it is $8 U / 9$. Three crosschannels, whose centers are located at the $x_{1}$ values of $l_{1}, l_{2}$ and $l_{3}$ and that have widths of $d_{1}, d_{2}$ and $d_{3}$ respectively, are to be used to cause velocity agitations that make the flow interface vary with time. Consonant with both experimental observations and theoretical considerations of no-slip boundary conditions $[158,157,159,161]$, we assume that the velocities in the crosschannels also satisfy parabolic conditions, leading to a velocity contribution

$$
v_{2}\left(x_{1}, t\right)=\frac{V_{j}}{d_{j}^{2}}\left[d_{j}^{2}-\left(x_{1}-l_{j}\right)^{2}\right] \phi_{j}(t), l_{j}-d_{j} \leq x_{1} \leq l_{j}+d_{j},(j=1,2,3),
$$

in the $x_{2}$ direction, where $V_{j}$ is the maximum velocity in the cross-channel, and $\phi_{j}(t)$ is the time-varying modulation that is being applied. To emphasize that neither GLCS nor LCS approaches require time-periodicity, we use the parameter values $L=1, U=1,\left\{V_{j}\right\}=\{0.3,0.2,0.05\},\left\{l_{j}\right\}=\{3,5,8\}$, $\left\{d_{j}\right\}=\{0.1,0.3,0.1\}$, and choose the functional forms

$$
\left\{\phi_{j}(t)\right\}=\{\cos (3 t), \tanh (t-5), \sin (\sqrt{2} t)\} .
$$

Thus, the total velocity in the channel is $v=\left(v_{1}, v_{2}\right)$, where $v_{1,2}$ are defined in (20) and (21). 


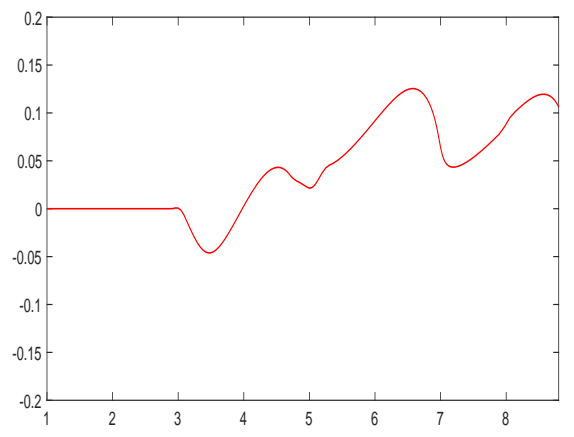

(a) Immiscible GLCS

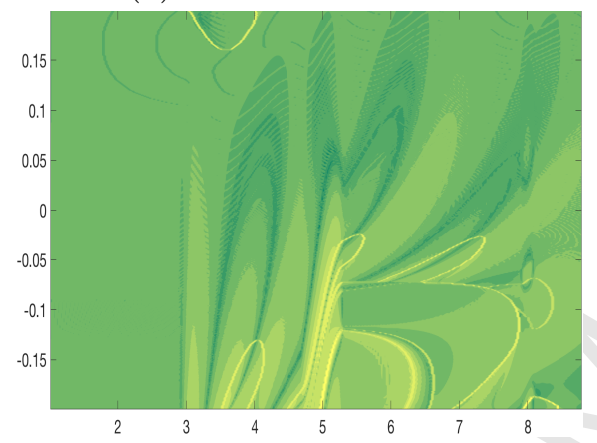

(c) FTLE field

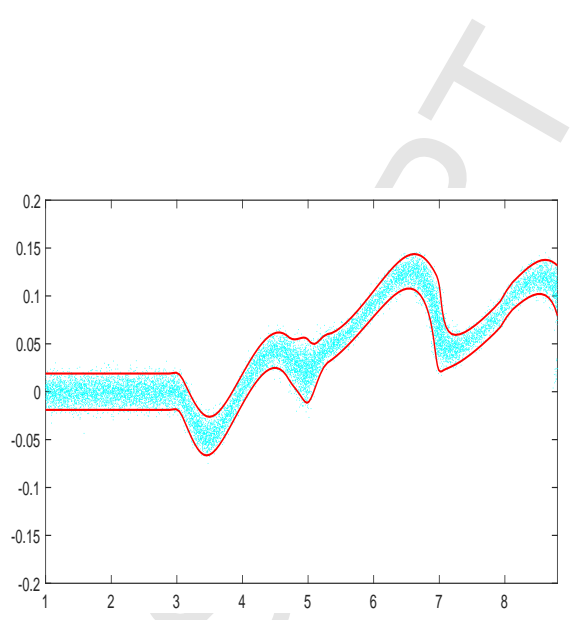

(b) Miscible GLCS

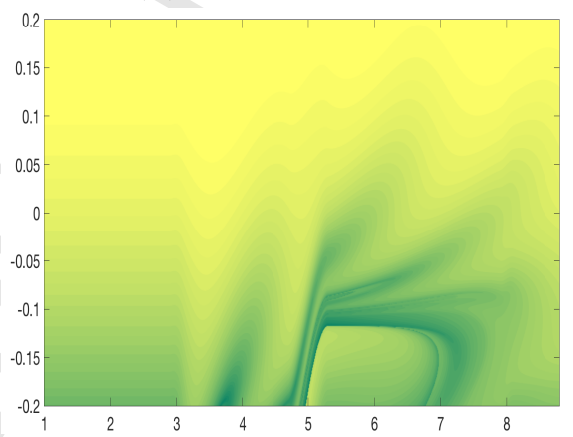

(d) Lagrangian speed average

Figure 8: Comparison between (a) immiscible and (b) miscible GLCSs at $t=10$ with diagnostic fields associated with LCS detection of the flow from time $t=10$ to $\tau=0$ : (c) backward FTLE field, and (d) Lagrangian average of speed.

Our GLCS will be the flow interface between the fluids at time $t=10$. Within the framework of our GLCS definition (11), we can think of determining this by implementing (13) with an initial uniform density $c_{0}$ supported on the upper fluid towards the left of the first cross-channel. If the fluids are immiscible, they will not mix, and this is equivalent to choosing $\mathrm{Pe}=\infty$. Then, when the density field has evolved to time 10, there would be a sharp transition in the density across a flow interface, which would be identifiable as a curve which we shall call the 'immiscible GLCS.' If the fluids were miscible, then $\mathrm{Pe}<\infty$, and the 'flow interface' (the 'miscible GLCS') would be more difficult to define. In this section, we show how the immiscible and miscible flow interfaces can be determined without having to numerically evolve (13), but by using alternative strategies.

Suppose first that $\mathrm{Pe}=\infty$ (the fluids do not mix). Because we have 
a priori knowledge that the interface is along the line $x_{2}=0$ for $x_{1}<$ $l_{1}-d_{1}$, we can simply generate the streakline emanating from the known flow interface $\left\{x_{2}=0, x_{1}<l_{1}-d_{1}\right\}$ (which could also be theoretically estimated if needed $[165,1])$. The GLCS obtained in this fashion at time 10 is shown in Fig. 8(a), and is a well-defined curve which separates the two fluids. On the other hand, let us suppose $\mathrm{Pe}<\infty$, and the fluids are miscible. In this case, an approach would be to consider the stochastic differential equation ${ }^{18}$ (16) with $\sigma=\mathrm{Id}$ and $\varepsilon=\sqrt{2 / \mathrm{Pe}}$, and consider the statistics at time 10 of particles lying on the curve $\mathcal{C} \equiv\left\{x_{2}=0, x_{1}<l_{1}-d_{1}\right\}$ at time 0 . It is known that, for small $\varepsilon$, the mean transverse deviation from the $\varepsilon=0$ flow separator (i.e., the immiscible GLCS of Fig. 8(a)), divided by $\varepsilon$, approaches zero [139]. Moreover, an explicit expression for the variance of displacement in the normal direction to the curve has been established (see equation (9) in [139]). The red curves in Fig. 8(b) are generated by this expression, going twice the standard deviation ${ }^{19}$ away from the immiscible interface, using $\varepsilon=0.003$. The region between the red curves is therefore a 'fuzzy flow interface' within which there is strong mixing between the upper and lower fluids, and this constitutes our 'miscible GLCS'. To demonstrate the validity of this theory, there are also $2 \times 10^{5}$ cyan dots plotted in this figure, which were generated by taking initial points on $\mathcal{C}$ and iterating them forward by (16) using the Euler-Maruyama scheme [166]. Their distribution seem to be captured nicely by the theoretical miscible GLCS, whose width varies along the channel. (There are ongoing extensions of using (16) in understanding coherence, including the idea of fuzzy stable/unstable manifolds [140] and using the variance as a global measure of uncertainty [141].)

For comparison, suppose we want to find an appropriate LCS at $t=10$; here, we think of LCS methods as being those which advect (1) directly thinking of all initial conditions as being equivalent. We show in Fig. 8(bc) several LCS diagnostic fields associated with the flow from time 10 to 0 . While we have not extracted any LCS sets from these fields, it is clear that they are not in any way connected to the GLCS of interest here. This is no surprise; the GLCS has nothing to do with, say, regions of extremal

\footnotetext{
${ }^{18}$ This is equivalent to using an advection-diffusion type evolution equation for the density, because there is associated with (16) a Fokker-Planck equation which does precisely that $[140,141]$.

${ }^{19}$ Twice the standard deviation is used as a proxy for $95 \%$ of the data; this is however not actually correct because it is not necessarily true that the distribution is normal.
} 
stretching, or of identifying rotational motion, etc.

\subsection{Spreading of tracers in the ocean}

In this example, we examine the global sea surface temperature (SST) as our co-evolving variable, and compare with an LCS diagnostic as well as a newly proposed method which falls within our GLCS framework. We show in Fig. 9 the SST field on 5 April, 2015 in the Atlantic ocean off the coast of North America. A warm SST tongue (dark red filament) associated with the Gulf Stream current extends along the coast until roughly the latitude of Cape Hatteras, where the Gulf Stream separates from the coast and turns eastward, carrying the warm water tongue with it into the North Atlantic. In this Gulf Stream Extension region, the strong and narrow Gulf Stream loses its coherence, widens, slows down and starts to meander. Some of these meanders grow and detach from the current forming coherent mesoscale eddies called Gulf Stream rings, which can transport warmer/colder water masses westward through the colder/warmer ambient water on the northern/southern side of the Gulf Stream Extension current. Fig. 9 shows such structures, for example, a cold-water (yellow) meander protruding southward into the (red) warmer waters near $58 \mathrm{~W}, 39 \mathrm{~N}$, a detatched (yellow) cold-core ring about 5 deg to the west from the meander, as well as a (yellow) cold-core ring further to the east, centered at about $45 \mathrm{~W}, 37 \mathrm{~N}$, which is surrounded by (orange) warmer waters.

The fact that one can easily tease out from the observed SST images signatures of well-known oceanographic currents, such as the Gulf Stream current with its meanders and eddies (satellite-based geostrophic velocities are shown by arrows in Fig. 10 (top left)), suggests that at meso- and larger spatial scales the evolution of SST is dominated by advection, rather than diffusion. Combined with the existence of the global ambient meridional SST gradient, this implies that the GLCS relevant for the SST should be similar to LCS. This general inference is confirmed in our numerical calculations of FTLEs based on ocean velocities inferred from satellite altimetry Fig. 10 (top right)). These calculations are based on geostrophic velocity fields $v$ inferred from AVISO satellite sea surface height observations. Although the FTLE field is quite complicated in this region, and its maximizing ridges, which serve as proxies for LCSs, form a complex tangled web, all three strong and distinct SST features described above - the southward-protruding meander, the cold-core ring to the west of it and the larger cold-core ring further eastare clearly visible in the concurrent FTLE snapshot. The FTLE values are 


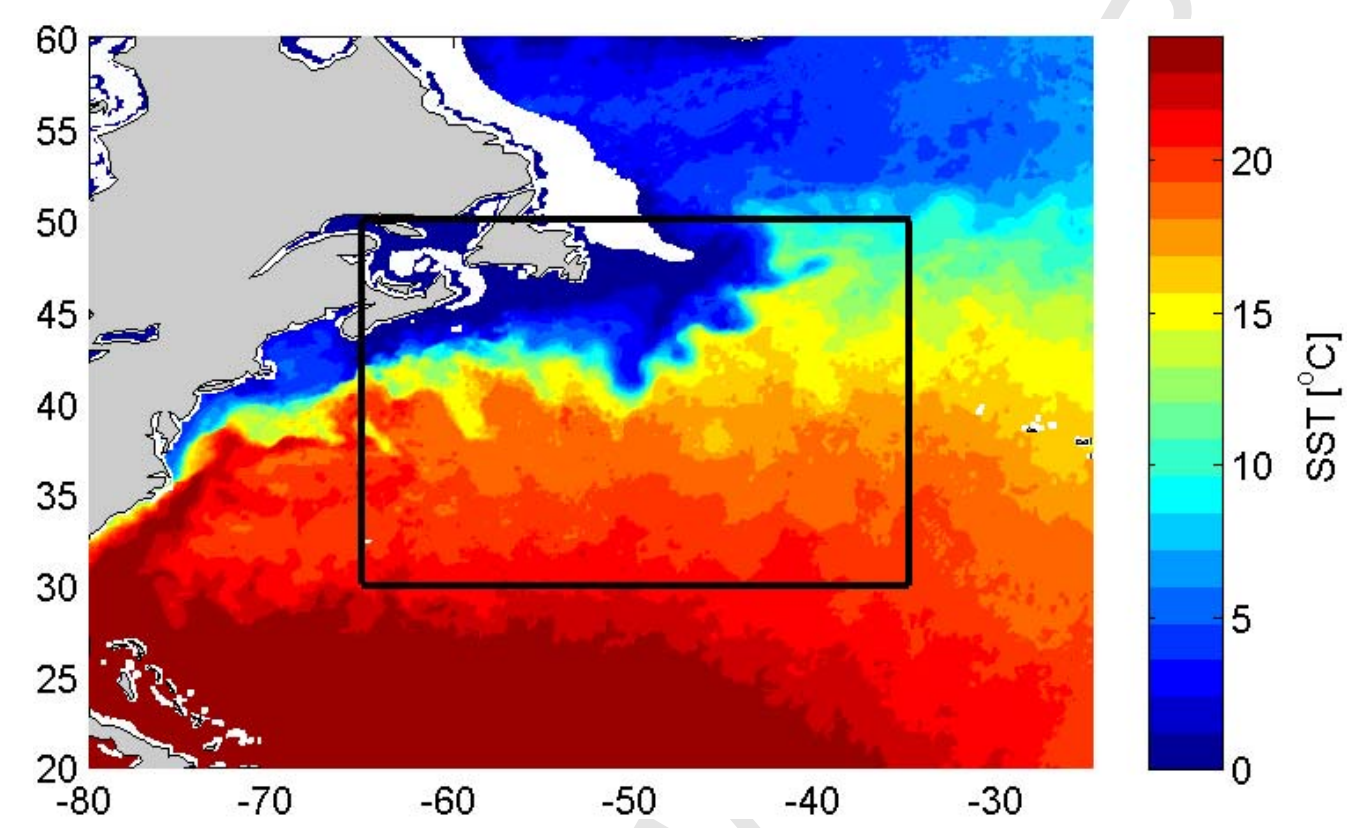

Figure 9: Satellite-based SST map on 5 Apr, 2015. Data was obtained from the Remote Sensing Systems group website (http://www.remss.com/measurements/sea-surfacetemperature) and corresponds to an optimally interpolated SST product that merged multiple satellites into globally complete gridded maps. The black box corresponds to the domain shown in Fig. 10.

generally larger at the flanks of the meandering Gulf Stream Extension Current than further from it, suggesting (correctly) that a tracer (such as SST) will likely experience stronger filamentation there. Of course, one cannot completely reproduce or infer the tracer distribution just based on FTLEs alone, without any information about the initial conditions; but nevertheless FTLEs are helpful in predicting and interpreting some of the dominant features and characteristics of a tracer distribution for a large-scale nonuniformly distributed advection-dominated tracer such as SST.

The situation is strikingly different for a tracer with a more localized initial distribution, as illustrated in Fig. 10 (top left). In this numerical simulation, we started with a Gaussian patch of elevated tracer concentration, which was centered at $52 \mathrm{~W}, 39 \mathrm{~N}$ and had $\sigma=3 \mathrm{deg}$ in both zonal and meridional directions, on top of the uniform ambient background concentration, $c_{b}=1$, everywhere else. Such a distribution might be relevant, for example, for a spill of a chemical pollutant into the ocean. The initial tracer distri- 
bution was then advected numerically using the AVISO-based geostrophic velocities $v$ without any explicit diffusion added, and it evolved, after 30 days, into the distribution shown in Fig. 10 (bottom left). Although the final tracer field bears some resemblance to the backward FTLEs shown in the top right panel, FTLEs fail to correctly predict even such dominant tracer features as the two arms of the tracer filaments in the east, the overall extent of the elevated tracer anomalies, absence of the elevated tracer concentrations to the west from the initial Gaussian blob, as well as the various hot spots of the tracer concentration, which do not always align well with the highest/sharpest FTLE ridges. To this end, we conclude that FTLEs - or, more generally, any LCSs - are of limited use in predicting and interpreting the spread of tracers in the ocean, even for advection- rather than diffusiondominated tracers, because LCS methods are not designed to take into account nonuniform initial distributions.

\subsubsection{GLCSs using u-star approach}

We now demonstrate one method for GLCS identification based on recent work by $[133,101]$ which builds on earlier work on heat transport by $[127$, $128,129,130,131,132]$. We show that when applied to the example in Fig. 10, the resulting GLCSs are in better agreement with the tracer than LCSs.

The u-star approach of [133] considers a tracer that evolves according to the transport equation (17). Following [127, 128, 129, 130, 131, 132], they define an effective velocity field $u^{*}$, in which the flux of a tracer is totally advective, i.e., $\Phi=c u^{*}$ [133]. Integrating the transport equation (17) over a material volume $V$ indicates that the amount of tracer inside any material volume $V$ advected by $u^{*}$ is conserved, because of the absence of sources or sinks. Thus, by tracing the evolution of material contours in $u^{*}$ we can make inferences about the spreading of a tracer. For example, if the invariant stable and unstable manifolds exist in the $u^{*}$ flow field, they represent transport barriers for the $u^{*}$-flow, and thus the amount of tracer should be conserved within the lobes between these barriers, allowing us to predict and interpret the process of tracer filamentation. In other words, LCS of $u^{*}$ are GLCS relevant for tracer $c$. In choosing an appropriate $u^{*}$, the fact that the flux can be augmented with any non-divergent vector field without changing the transport equation (17) has previously been noted in the 'heatline' approaches $[132,129]$, and a deviation from a physically-relevant state has 

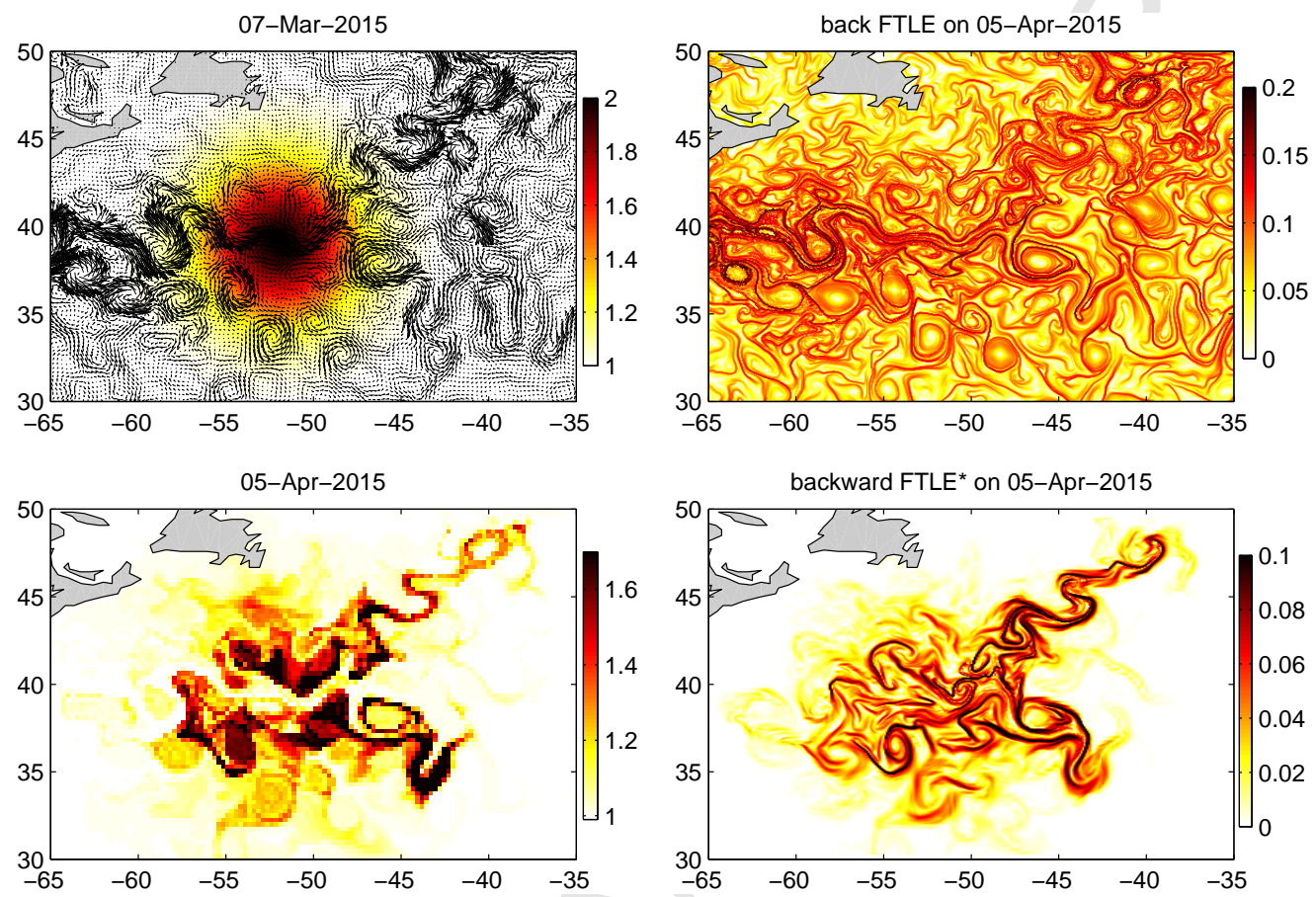

Figure 10: Initial (4 Mar, 2015 - top left) and final (5 Apr, 2015 - bottom left) tracer field, and the corresponding backward FTLE (top right) and FTLE* (bottom right) fields on 5 Apr, 2015. Calculations are based on AVISO gridded maps of absolute dynamic topography (i.e., on geostrophic velocities) inferred from satellite sea surface height observations.

been used. The suggested formulation in [133] is to use $\mathrm{e}^{20}$

$$
u^{*}(x, y, t)=\frac{\Phi}{c(x, y, t)}=v(x, y, t)\left(1-\frac{c_{b}}{c(x, y, t)}\right)
$$

in which $v$ is the AVISO-obtained velocity field, and $c$ the tracer density, at locations $(x, y)$ and time $t$, and $c_{b}=1$ is the background value of the tracer density. Given $u^{*}$ as in (22), any method of LCS identification can be applied to it. Here, we demonstrate the method using Lyapunov exponents, which are arguably the most used LCS method in oceanography. FTLEs computed using $u^{*}$ will be denoted FTLE* ${ }^{*}$ In a way, this approach is similar in spirit

\footnotetext{
${ }^{20}$ This identifies the flux $\Phi:=v\left(c-c_{b}\right)$ for usage in the transport equation (17), with $c-c_{b}$ capturing the deviation, as opposed to generic advection in incompressible situations which would have $\Phi=c v$.
} 
to that by [114], who estimated GLCSs (although the authors do not use this terminology) for actively swimming particles by computing FTLEs for a modified velocity field that was a sum of fluid and swimming velocities.

The bottom right panel of Fig. 10 shows the backward FTLE* field, which was estimated numerically from grids of trajectories advected (in backward time) by the numerically-constructed $u^{*}$, which in turn was computed using eq. (22) from the numerically-computed tracer concentrations. Maximizing ridges of FTLE*, which define tracer-relevant GLCS, are in a better agreement with the final tracer distribution than FTLE ridges. Several features are correctly captured by FTLE* that were not captured by FTLE. First, the two arms of elevated tracer anomaly filaments align well with the strong FTLE* ${ }^{*}$ ridges in the eastern part of the distribution. Second, the overall shape and extent of the elevated tracer anomaly is confined to the area of elevated FTLE* ${ }^{*}$ Third, most of the dominant hot spots of the tracer anomaly agree fairly well with the height and sharpness of the FTLE* ridges, although the agreement is not perfect. For example, the white ribbon cutting through the tracer distribution near the Gulf Stream core is not captured by the FTLE* field. Thus, this method is not definitive, with clear scope for improvement and/or development of other methods for addressing structures in evolving density fields.

In summary, although LCSs have some value in predicting the spread of tracers in the ocean, they should be used with extreme caution, especially in problems involving localized tracer distributions, even for conservative and advection-dominated tracers. Evolution of tracers that are subject to diffusion, have strong sources and sinks, and/or are non-conservative and decay in time, would be even more different from LCSs. Thus, GLCSs are generally required to describe the evolution of tracers in the ocean. One method for GLCS identification has been put forward that combines the well-known FTLE technique with the recently-developed $u^{*}$-approach. The resulting GLCSs are more relevant for tracers, and the method could be easily generalized to account for diffusion and some forms of source/sink terms.

\section{Concluding remarks}

The concept of Generalized Lagrangian Coherent Structures (GLCSs) was formulated in this article to capture important flow entities whose evolution is linked to an unsteady velocity field $v(x, t)$. This generalizes Lagrangian Coherent Structure (LCS) methods in which the evolution is specifically of 
trajectories of $\dot{x}=v(x, t)$. We have used the GLCS framework to encapsulate a variety of methods which have been suggested to capture spatial "regions of interest" and their time-evolution. Indeed, the presentation is consistent with "coherent structures" as defined in other disciplines, e.g., pattern formation in reaction-diffusion equations modeling biological phenomena.

We have argued that LCSs are a special case of GLCSs, but that in general, GLCSs and LCSs may be different. This is inevitable, since the GLCSs are associated with a variable (or variables) whose evolution may obey a law different from the trajectories of $\dot{x}=v(x, t)$. As such, any identified GLCSs will depend on the particular law, how it is coupled to the velocity field $v(x, t)$, as well as an initial condition associated with the variable of interest. We have illustrated these facts through numerical examples.

In addition to introducing the GLCS framework, we have discussed methods for LCS extraction in some detail. We have emphasized and illustrated the fact that different LCS methods themselves often give different 'results' (cf. Figs. 4 and 5), which is because they each have different criteria. Thus, we highlight the danger of reaching conclusions about properties which are not explicitly sought by a particular LCS method. Additionally, we have in Section 2.3 discussed a range of aspects that must be borne in mind when using LCS diagnostics: the fact that LCSs are wedded to the time-interval chosen, whether it is justifiable to think of the LCS structures as appropriately time-parametrized, whether the LCSs are advected materially, whether the diagnostic procedure is frame-independent, whether there is implied timeperiodicity when a genuinely finite-time approach is required, and the fact that each LCS procedure inevitably uses some tolerances/conditions in its numerical operation. While many studies apply an LCS method to determine 'nice' LCS structures, all these facts must be considered in evaluating the suitability of the approach. And in particular, speculative conclusions regarding other aspects not explicitly targeted by the LCS approach must be avoided. This is certainly true of properties which we have labelled 'GLCSs,' in which co-evolving variables not necessarily addressed by an LCS method are of interest.

One type of co-evolved variable of interest would be determining the distribution of a scalar field for which an advective flux can be constructed from the velocity field according to the transport equation (17). This forms an important class of GLCSs, which includes advection-diffusion of fields such as temperature or pollutant concentration. However, we have characterized GLCSs as being associated with variables whose evolution is not necessarily 
confined to a governing law (17), but is subject to a more general evolution.

A particularly interesting connection between GLCSs and LCSs arises in transfer operator methods, which as we shall now argue straddles the boundary between the methods. In the standard ODE implementation of transfer operators, (1) is used as the sole evolution equation, to help determine the transfer operator numerically. All initial conditions are considered equal in this approach. However, the transfer operator is actually associated with a PDE - the Fokker-Planck equation - which is an advection-diffusion equation of the form (13). There are also subtle issues as to how this PDE, with its diffusion term, relates to the ODE; this is associated with the singular limit $\mathrm{Pe} \rightarrow \infty$ as opposed to simply setting $\mathrm{Pe}=\infty$ (see $[120,88]$ ). If using evolution of this PDE, an initial density distribution must be specified; this provides a weighting of $x$ at the initial time which does not happen for the ODE implementation. Using the PDE in this fashion would classify this as a GLCS approach as opposed to an LCS one. Commonly, however, the PDE approach is not what is used in extracting coherent structures, but rather the ODE with unweighted initial conditions. The transfer operator then helps determine forms of $c_{0}$ satisfying 'almost invariant' propensities, as opposed to having $c_{0}$ specified as in our GLCS framework.

We have presented GLCSs as a useful umbrella for reviewing a range of methods which continue to be developed for understanding coherent structures. In particular, GLCSs form an excellent framework for reviewing "standard" LCS methods (associated solely with $\dot{x}=v(x, t)$ ), as well as "extended" methods that target variables whose evolution is linked to $v$. In reviewing these methods, we have broadly discussed both theoretical and applied issues associated with each method.

Acknowledgments: SB acknowledges with thanks support from the Australian Research Council through Future Fellowship grant FT130100484 and Discovery Project grant DP170100277. NTO acknowledges support from the U.S. National Science Foundation under grant CMMI-1563489. IR would like to acknowledge support from the U.S. NSF grant OCE-1558806, ONR grant N00014-11-10087 and NASA grant NNX14AH29G. SB and NTO also express thanks to the Banff International Research Station who supported a workshop on "Transport in unsteady flows: from deterministic structures to stochastic models and back again" in January 2017 [134], which helped highlight the necessity for disseminating the role of LCSs in flows, in particular what they do and do not tell us. Detailed comments from anonymous ref- 
erees are also gratefully acknowledged; these have helped refine this review paper at both conceptual and detailed levels.

[1] S. Balasuriya, Barriers and transport in unsteady flows: a Melnikov approach, Series on Mathematical Modeling and Computation, SIAM Press, Philadelphia, 2016.

[2] J. Guckenheimer, P. Holmes, Nonlinear oscillations, dynamical systems, and bifurcations of vector fields, Springer, New York, 1983.

[3] F. Beron-Vera, M. Olascoaga, M. Brown, H. Kocak, I. Rypina, Invariant-tori-like Lagrangian coherent structures in geophysical flows, Chaos 20 (2010) 017514.

[4] N. T. Ouellette, On the dynamical role of coherent structures in turbulence, C. R. Physique 13 (2012) 866-877.

[5] G. Haller, G.-C. Yuan, Lagrangian coherent structures and mixing in two-dimensional turbulence, Phys. D 147 (2000) 353-370.

[6] G. Haller, Distinguished material surfaces and coherent structures in three-dimensional fluid flows, Phys. D 149 (2001) 248-277.

[7] G. Haller, Lagrangian coherent structures from approximate velocity data, Phys. Fluids A 14 (2002) 1851-1861.

[8] M. Serra, G. Haller, Objective Eulerian coherent structures, Chaos 26 (2016) 053110.

[9] G. Haller, Lagrangian Coherent Structures, Annu. Rev. Fluid Mech. 47 (2015) 137-162.

[10] R. Samelson, Lagrangian motion, coherent structures, and lines of persistent material strain, Annu. Rev. Marine Sci. 5 (2013) 137-163.

[11] S. Shadden, Lagrangian coherent structures, in: R. Grigoriev (Ed.), Transport and Mixing in Laminar Flows: From Microfluidics to Oceanic Currents, Wiley, Berlin, 2011, pp. 59-89.

[12] T. Peacock, G. Haller, Lagrangian coherent structures: the hidden skeleton of fluid flow, Phys. Today 66 (2013) 41-47. 
[13] G. Boffetta, G. Lacorata, G. Radaelli, A. Vulpiani, Detecting barriers to transport: a review of different techniques, Phys. D 159 (2001) 5870 .

[14] A. Okubo, Horizontal dispersion of floatable particles in the vicinity of velocity singularities such as convergences, Deep Sea Res. 17 (1970) $445-454$

[15] J. Weiss, The dynamics of enstrophy transfer in two-dimensional hydrodynamics, Phys. D 48 (1991) 273-294.

[16] M. Chong, A. Perry, B. Cantwell, A general classification of threedimensional flow fields, Phys. Fluids A 2 (1990) 765-777.

[17] J. C. R. Hunt, A. Wray, P. Moin, Eddies, stream, and convergence zones in turbulent flows, Tech. Rep. CTR-S88, Center for Turbulence Research (1988).

[18] C. Basdevant, T. Philipovitch, On the validity of the "Weiss criterion" in two-dimensional turbulence, Physica D 73 (1994) 17-30.

[19] J. Jeong, F. Hussain, On the identification of a vortex, J. Fluid Mech. 285 (1995) 69-94.

[20] B. L. Hua, P. Klein, An exact criterion for the stirring properties of nearly two-dimensional turbulence, Physica D 113 (1998) 98-110.

[21] W. A. Coppel, Dichotomies in Stability Theory, no. 629 in Lecture Notes in Mathematics, Springer-Verlag, Berlin, 1978.

[22] F. Battelli, C. Lazzari, Exponential dichotomies, heteroclinic orbits and Melnikov functions, J. Differential Equations 86 (1986) 342-366.

[23] K. Palmer, Exponential dichotomies and transversal homoclinic points, J. Differential Equations 55 (1984) 225-256.

[24] S. Balasuriya, Explicit invariant manifolds and specialised trajectories in a class of unsteady flows, Phys. Fluids 24 (2012) 127101.

[25] R. Samelson, S. Wiggins, Lagrangian transport in geophysical jets and waves, Interdisciplinary Applied Mathematics, Springer, 2006. 
[26] V. Rom-Kedar, A. Leonard, S. Wiggins, An analytical study of transport, mixing and chaos in an unsteady vortical flow, J. Fluid Mech. 214 (1990) 347-394.

[27] S. Wiggins, Chaotic transport in Dynamical Systems, Springer, New York, 1992.

[28] V. Rom-Kedar, S. Wiggins, Transport in two-dimensional maps, Arch. Ration. Mech. Anal. 109 (1990) 239-298.

[29] K. Mitchell, Partitioning two-dimensional mixed phase spaces, Phys. D 241 (2012) 1718-1734.

[30] S. Balasuriya, Cross-separatrix flux in time-aperiodic and timeimpulsive flows, Nonlinearity 19 (2006) 2775-2795.

[31] S. Balasuriya, A tangential displacement theory for locating perturbed saddles and their manifolds, SIAM J. Appl. Dyn. Sys. 10 (2011) 11001126 .

[32] J. Mahoney, K. Mitchell, Finite-time barriers to front propagation in two-dimensional fluid flows, Chaos 25 (2015) 087404.

[33] J. Mahoney, J. Li, C. Boyer, T. Solomon, K. Mitchell, Frozen reaction fronts in steady flows: A burning-invariant-manifold perspective, Phys. Rev. E 92 (2015) 063005.

[34] J. Mahoney, D. Bergteil, M. Kingsbury, K. Mitchell, T. Solomon, Invariant barriers to reactive front propagation in fluid flows, Europhys. Lett. 98 (2012) 44005.

[35] P. Megson, M. Najarian, K. Lilienthal, T. Solomon, Pinning of reaction fronts by burning invariant manifolds in extended flows, Phys. Fluids 27 (2015) 023601.

[36] S. Gowen, T. Solomon, Experimental studies of coherent structures in an advection-reactiondiffusion system, Chaos 25 (2015) 087403.

[37] N. Santitissadeekorn, D. Bohl, E. Bollt, Analysis and modeling of an experimental device by finite-time Lyapunov exponent method, Intern. J. Bifurc. Chaos 19 (2009) 993-1006. 
[38] G. Rosi, A. Walker, D. Rival, Lagrangian coherent structure identification using a Voronoi tessellation-based networking algorithm, Exp. Fluids 56 (2015) 189.

[39] M. Green, C. Rowley, A. Smits, The unsteady three-dimensional wake produced by a trapezoidal pitching panel, J. Fluid Mech. 685 (2011) $117-145$.

[40] D. Kelley, M. Allshouse, N. Ouellette, Lagrangian coherent structures separate dynamically distinct regions in fluid flow, Phys. Rev. E 88 (2013) 013017.

[41] B. Rutherford, T. Dunkerton, M. Montgomery, Lagrangian vortices in developing tropical cyclones, Q. J. R. Meteorol. Soc. 141 (2015) 33443354 .

[42] C. Harrison, G. Glatzmaier, Lagrangian coherent structures in the California current system: sensitiivities and limitations, Geophys. Astrophys. Fluid Dyn. 106 (2012) 22-44.

[43] S. Shadden, F. Lekien, J. Paduan, F. Chavez, J. Marsden, The correlation between surface drifters and coherent structures based on highfrequency radar data in Monterey bay, Deep-Sea Res. II 56 (2009) $161-172$.

[44] L. Gaultier, J. Verron, J.-M. Brankart, O. Titaud, P. Brasseur, On the inversion of submesoscale tracer fields to estimate the surface ocean circulation, J. Marine Sys. 126 (2013) 33-42.

[45] L. Gaultier, B. Djath, J. Verron, J. Brankart, P. Brasseur, A. Melet, Inversion of submesoscale patterns from a high-resolution Solomon Sea model: Feasibility assessment, J. Geophys. Res. Oceans 119 (2014) $4520-4541$.

[46] I. Sala, C. Harrison, R. Caldeira, The role of the Azores archipelago in capturing and retaining incoming particles, J. Marine Sys. 154 (2016) $146-156$.

[47] B. Knutson, W. Tang, P. Chan, Lagrangian coherent structure analysis of terminal winds: three-dimensionality, intramodel variations and flight analyses, Adv. Meteor. 2015 (2015) 816727. 
[48] H. Guo, W. He, T. Peterka, H.-W. Shen, S. Collis, J. Helmus, Finitetime Lyapunov exponents and Lagrangian coherent structures in uncertain unsteady flows, IEEE Trans. Visual. Comp. Graphics 22 (2016) $1672-1682$.

[49] A. BozorgMagham, S. Ross, D. Schmale, Real-time prediction of atmospheric Lagrangian coherent structures based on forecast data: an application and error analysis, Phys. D 258 (2013) 47-60.

[50] I. Rypina, S. Scott, L. Pratt, M. Brown, Investigating the connection between complexity of isolated trajectories and Lagrangian coherent structures, Nonlin. Proc. Geophys. 18 (2011) 977-987.

[51] I. Rypina, L. Pratt, J. Pullen, J. Levin, A. Gordon, Chaotic advection in an archipelago, J. Phys. Oceanography 40 (2010) 1988-2006.

[52] I. Rypina, M. Brown, H. Kocak, Transport in an idealized three-gyre system with application to the Adriatic Sea, J. Phys. Oceanography 39 (2009) 675-690.

[53] I. Rypina, L. Pratt, S. Lozier, Near-surface transport pathways in the North Atlantic ocean, J. Phys. Oceanography 41 (2011) 911-925.

[54] D. Acheson, Elementary Fluid Dynamics, Oxford University Press, 1990.

[55] L. Landau, E. Lifshitz, Fluid mechanics, Pergamon, London, 1958.

[56] A. Luttman, E. Bollt, R. Basnayake, S. Kramer, A stream function approach to optical flow with applications to fluid transport dynamics, Proc. Appl. Math. Mech. 11 (2011) 855-856.

[57] S. Balasuriya, Impulsive perturbations to differential equations: stable/unstable pseudo-manifolds, heteroclinic connections, and flux, Nonlinearity 29 (2016) 3897-3933.

[58] G. Eyink, Locality of turbulent cascades, Phys. D 207 (2005) 91-116.

[59] K. Ide, D. Small, S. Wiggins, Distinguished hyperbolic trajectories in time dependent fluid flows: Analytical and computational approach for velocity fields defined as data sets, Nonlin. Proc. Geophys. 9 (2002) $237-263$. 
[60] A. Mancho, S. Wiggins, J. Curbelo, C. Mendoza, Lagrangian descriptors: A method for revealing phase space structures of general time dependent dynamical systems, Commun. Nonlin. Sci. Numer. Simu. 18 (2013) 3530-3557.

[61] I. Mezić, S. Loire, V. Fonoberov, P. Hogan, A new mixing diagnostic and Gulf oil spill movement, Science 330 (2010) 486-489.

[62] M. Budišić, S. Siegmund, T. Doan, I. Mezić, Mesochronic classification of trajectories in incompressible $3 \mathrm{~d}$ vector fields over finite times, Discrete Cont. Dyn. Sys. S 9 (2016) 923 - 958.

[63] G. Haller, A. Hadjighasem, M. Farazmand, F. Huhn, Defining coherent vortices objectively from the vorticity, J. Fluid Mech. 795 (2016) 136173.

[64] S. Scott, T. Redd, L. Kuznetsov, I. Mezić, C. Jones, Capturing deviation from ergodicity at different scales, Phys. D 238 (2009) 1668-1679.

[65] D. Elhmaïdi, A. Provenzale, A. Babiano, Elementrary topology of two-dimensional turbulence from a Lagrangian viewpoint and singleparticle dispersion, J. Fluid Mech. 257 (1993) 533-558.

[66] S. Balasuriya, Local stable and unstable manifolds and their control in nonautonomous finite-time flows, J. Nonlin. Sci. 26 (2016) 895-927.

[67] B. Sandstede, S. Balasuriya, C. Jones, P. Miller, Melnikov theory for finite-time vector fields, Nonlinearity 13 (2000) 1357-1377.

[68] M. Farazmand, G. Haller, Computing Lagrangian Coherent Structures from variational LCS theory, Chaos 22 (2012) 013128.

[69] G. Haller, F. Beron-Vera, Geodesic theory for transport barriers in two-dimensional flows, Phys. D 241 (2012) 1680-1702.

[70] K. Onu, F. Huhn, G. Haller, LCS Tool: an algorithmic introduction to Lagrangian Coherent Structures, J. Comp. Sci. 7 (2015) 26-36.

[71] D. Blazevski, G. Haller, Hyperbolic and elliptic transport barriers in three-dimensional unsteady flows, Phys. D 273-274 (2014) 46-64. 
[72] H. Teramoto, G. Haller, T. Komatsuzaki, Detecting invariant manifolds as stationary LCSs in autonomous dynamical systems, Chaos 23 (2013) 043107.

[73] R. Pierrehumbert, H. Yang, Global chaotic mixing in isentropic surfaces, J. Atmospheric Sci. 50 (1993) 2462-2480.

[74] G. Voth, G. Haller, J. Gollub, Experimental measurements of stretching fields in fluid mixing, Phys. Rev. Lett. 88 (2002) 254501.

[75] S. Shadden, F. Lekien, J. Marsden, Definition and properties of Lagrangian coherent structures from finite-time Lyapunov exponents in two-dimensional aperiodic flows, Phys. D 212 (2005) 271-304.

[76] P. Tallapragada, S. Ross, A set oriented definition of finite-time Lyapunov exponents and coherent sets, Commun. Nonlin. Sci. Numer. Simu. 18 (2013) 1106-1126.

[77] K. McIlhany, S. Wiggins, Eulerian indicators under continuously varying conditions, Phys. Fluids 24 (2012) 073601.

[78] S. Brunton, C. Rowley, Fast computation of finite-time Lyapunov exponent fields for unsteady flows, Chaos 20 (2010) 017503.

[79] D. Lipinski, K. Mohseni, A ridge tracking algorithm and error estimate for efficient computation of Lagrangian coherent structures, Chaos 20 (2010) 017504.

[80] R. Horn, C. Johnson, Matrix analysis, Cambridge University Press, Cambridge, 1999.

[81] G. Haller, A variational theory for Lagrangian Coherent Structures, Phys. D 240 (2011) 574-598.

[82] S. Balasuriya, R. Kalampattel, N. Ouellette, Hyperbolic neighborhoods as organizers of finite-time exponential stretching, J. Fluid Mech. 807 (2016) 509-545.

[83] F. d'Ovidio, V. Fernández, E. Hérnandez-Garcia, C. López, Mixing structures in the Mediterranean sea from finite size Lyapunov exponents, Geophys. Res. Lett. 31 (2004) L17203. 
[84] F. d'Ovidio, S. D. Monte, S. Alvain, Y. Dandonneau, M. Lévy, Fluid dynamical niches of phytoplankton types, Proc. Natl. Acad. Sci. USA 107 (2010) 18366-18370.

[85] F. d'Ovidio, S. D. Monte, A. Penna, C. Cotté, C. Guinet, Ecological implications of eddy retentionin the open ocean: a Lagrangian approach, J. Phys. A: Math. Theor. 46 (2013) 254023.

[86] D. Karrasch, G. Haller, Do Finite-Size Lyapunov Exponents detect coherent structures?, Chaos 23 (2013) 043126.

[87] M. Dellnitz, O. Junge, Set-oriented methods for dynamical systems, in: B. Fiedler, G. Iooss, N. Kopell (Eds.), Handbook of Dynamical Systems III: Towards Applications, World Scientific, 2002.

[88] G. Froyland, An analytic framework for identifying finite-time coherent sets in time-dependent dynamical systems, Phys. D 250 (2013) 1-19.

[89] G. Froyland, K. Padberg, Almost invariant sets and invariant manifolds: connecting probablistic and geometric descriptions of coherent structures in flows, Phys. D 238 (2009) 1507-1523.

[90] G. Froyland, N. Santitissadeekorn, A. Monahan, Transport in timedependent dynamical systems: Finite-time coherent sets, Chaos 20 (2010) 043116.

[91] T. Ma, E. Bollt, Relatively coherent sets as a hierarchical partition method, Intern. J. Bifurc. Chaos 23 (2013) 1330026.

[92] M. Allshouse, J.-L. Thiffeault, Detecting coherent structures using braids, Phys. D 241 (2012) 95-105.

[93] M. Budisić, J.-L. Thiffeault, Finite-time braiding exponents, Chaos 25 (2015) 087407.

[94] P. Grover, S. Ross, M. Stremler, P. Kumar, Topological chaos, braiding and bifurcation of almost-cyclic sets, Chaos 22 (2012) 043135.

[95] M. Stremler, S. Ross, P. Grover, P. Kumar, Topological chaos and periodic braiding of almost-cyclic sets, Phys. Rev. Lett. 106 (2011) 114101. 
[96] G. Froyland, K. Padberg-Gehle, A rough-and-ready cluster-based approach for extracting finite-time coherent sets from sparse and incomplete trajectory data, Chaos 25 (2015) 087406.

[97] A. Hadjighasem, D. Karrasch, H. Teramoto, G. Haller, Spectral clustering approach to Lagrangian vortex detection, Phys. Rev. E 93 (2016) 063107.

[98] K. Schlueter-Kuck, J. Dabiri, Coherent structure coloring: identification of coherent structures from sparse data using graph theory, J. Fluid Mech. 811 (2017) 468-486.

[99] K. Padberg-Gehle, C. Schneide, Network-based study of Lagrangian transport and mixing, Nonlin. Proc. Geophys. 24 (2017) 661-671.

[100] I. Rypina, L. Pratt, Trajectory encounter number as a diagnostic of mixing potential in fluid flows, Nonlin. Proc. Geophys. 24 (2017) 189202.

[101] I. Rypina, S. L. Smith, L. Pratt, Trajectory encounter number as a diagnostic of mixing potential in fluid flows: connections to diffusivity, Nonlin. Proc. Geophys. under review.

[102] T. Ma, E. Bollt, Differential geometry perspective of shape coherence and curvature evolution by finite-time nonhyperbolic splitting, SIAM J. Appl. Dyn. Sys. 13 (2014) 1106-1136.

[103] R. Mundel, E. Fredj, H. Gildor, V. Rom-Kedar, New Lagrangian diagnostics for characterizing fluid flow mixing, Phys. Fluids 26 (2014) 26602.

[104] G. Froyland, K. Padberg-Gehle, Finite-time entropy: a probabilistic method for measuring nonlinear stretching, Phys. D 241 (2012) 16121628 .

[105] S. Balasuriya, G. Froyland, N. Santitissadeekorn, Absolute flux optimising curves of flows on a surface, J. Math. Anal. Appl. 409 (2014) 119-139.

[106] M. Budišić, I. Mezić, Geometry of ergodic quotient reveals coherent structures in flows, Phys. D 241 (2012) 1255-1269. 
[107] N. Malhotra, I. Mezić, S. Wiggins, Patchiness: a new diagnostic for Lagrangian trajectory analysis in time-dependent flows, Intern. J. Bifurc. Chaos 8 (1998) 1053-1093.

[108] T. Ma, N. Ouellette, E. Bollt, Stretching and folding in finite time, Chaos 26 (2016) 023112.

[109] M. Williams, I. Rypina, C. Rowley, Identifying finite-time coherent sets from limited quantities of Lagrangian data, Chaos 25 (2015) 087408.

[110] A. Hadjighasem, M. Farazmand, D. Blazevski, G. Froyland, G. Haller, A critical comparison of lagrangian methods for coherent structure detection, Chaos 27 (2017) 053104.

[111] T. Sapsis, G. Haller, Inertial particle dynamics in a hurricane, J. Atmos. Sci. 66 (2009) 2481-2492.

[112] T. Sapsis, G. Haller, Clustering criterion for inertial particles in twodimensional time-periodic and three-dimensional steady flows, Chaos 20 (2010) 017515.

[113] F. J. Beron-Vera, M. J. Olascoaga, G. Haller, M. Farazmand, J. Trinanes, Y. Wang, Dissipative inertial transport patterns near coherent Lagranigan eddies in the ocean, Chaos 25 (2015) 087412.

[114] J. Peng, J. Dabiri, Transport of inertial particles by Lagrangian coherent structures: application of predator-prey interaction to jellyfish feeding, J. Fluid Mech. 623 (2009) 75-84.

[115] R. Ni, S. Kramel, N. T. Ouellette, G. A. Voth, Measurements of the coupling between the tumbling of rods and the velocity gradient tensor in turbulence, J. Fluid Mech. 766 (2015) 202-225.

[116] G. Haller, Lagrangian coherent structures from approximate velocity data, Phys. Fluids 14 (2002) 1851-1861.

[117] D. Karrasch, Comment on "A variational theory of hyperbolic Lagrangian Coherent Structures, Physica D 240 (2011) 574-598, Phys. D 241 (2012) 1470-1473.

[118] J.-L. Thiffeault, Using multiscale norms to quantify mixing and transport, Nonlinearity 84 (2012) R1-R44. 
[119] K. Koshel, E. Ryzhov, V. Zhmur, Diffusion-affected passive scalar transport in an ellipsoidal vortex in shear flow, Nonlin. Proc. Geophys. 20 (2013) 437-444.

[120] G. Froyland, Dynamic isoperimetry and the geometry of Lagrangian coherent structures, Nonlinearity 28 (2015) 3587-3622.

[121] G. Froyland, E. Kwok, A dynamic Laplacian for identifying Lagrangian coherent structures on weighted Riemannian manifolds, J. Nonlin. Sci. (2018) in press.

[122] D. Karrasch, J. Keller, A geometric heat-fow theory of Lagrangian coherent structures, ArXiv (2017) arXiv:1608.05598v4.

[123] K. Mitchell, J. Mahoney, Invariant manifolds and the geometry of front propagation in fluid flows, Chaos 22 (2012) 037104.

[124] M. Maxey, J. Riley, Equation of motion for a small rigid sphere in a nonuniform flow, Phys. Fluids 26 (1983) 883-889.

[125] S. Sundaram, L. R. Collins, Collision statistics in an isotropic particleladen turbulent suspension. part 1. direct numerical simulations, J. Fluid Mech. 335 (1997) 75-109.

[126] G. Falkovich, A. Fouxon, M. G. Stepanov, Acceleration of rain initiation by cloud turbulence, Nature 419 (2002) 151-154.

[127] S. Kimura, A. Bejan, The "heatline" visualization of convective heat transfer, ASME J. Heat Transfer 105 (1983) 916-919.

[128] A. Bejan, Convection Heat Transfer, Wiley, New York, 1995.

[129] V. Costa, Bejan's heatlines and masslines for convection visualization and analysis, Appl. Mech. Rev. 59 (2006) 127.

[130] S. Mahmud, R. Fraser, Visualizing energy flows through energy streamlines and pathlines, Int. J. Heat Mass Transfer 50 (2007) 3990.

[131] A. Mukhopadhyay, X. Qin, S. Aggarwal, I. Puri, On extension of "heatline" and "massline" concepts to reacting flows through use of conserved scalars, ASME J. Heat Transfer 124 (2002) 791. 
[132] M. Speetjens, A generalised Lagrangian formalism for thermal analysis of laminar convective heat transfer, Int. J. Therm. Sci. 61 (2012) 79-93.

[133] L. Pratt, R. Barkan, I. Rypina, Scalar flux kinematics, Fluids 1 (2016) 27.

[134] S. Balasuriya, D. Crommelin, A. Monahan, G. Froyland, N. Ouellette, L. Zanna, Transport in unsteady flows: from deterministic structures to stochastic models and back again, in: BIRS Workshop Reports, Banff International Research Station, Banff, Canada, 2017, pp. January 1520 .

[135] J. LaCasce, Statistics from Lagrangian observations, Progress in Oceanography 77 (2008) 1-29.

[136] P. Berloff, Dynamically consistent parametrization of mesoscale eddiesPart II eddy fluxes and diffusivity from transient impulses, Fluids 1 (2016) 22.

[137] E. Hendricks, W. Schubert, Transport and mixing in idealized barotropic hurricane-like vortices, Q. J. R. Meteorol. Soc. 135 (2009) $1456-1470$.

[138] I. Kamenkovich, I. Rypina, P. Berloff, Properties and origins of the anisotropic eddy-induced transport in the North Atlantic, J. Phys. Oceanography 45 (2015) 778-791.

[139] S. Balasuriya, Stochastic uncertainty of advected curves in finite-time unsteady flows, Phys. Rev. E 95 (2017) 062201.

[140] S. Balasuriya, G. Gottwald, Stochastic perturbations of stable/unstable manifolds, with implications to transport, Phys. D (2017) submitted.

[141] S. Balasuriya, Stochastic sensitivity: a computable measure for uncertainty of deterministic trajectories, Annal. Appl. Prob. (2017) submitted.

[142] K. Priyankara, S. Balasuriya, E. Bollt, Quantifying the role of folding in nonautonomous flows: the unsteady double gyre, Intern. J. Bifurc. Chaos 27 (2017) 1750156. 
[143] M. Allshouse, T. Peacock, Lagrangian based methods for coherent structure detection, Chaos 25 (2015) 097617.

[144] K. Pratt, J. Meiss, J. Crimaldi, Reaction enhancement of initially distant scalars by Lagrangian coherent structures, Phys. Fluids 27 (2015) 035106.

[145] M. Sudharsan, S. Brunton, J. Riley, Lagrangian coherent structures and inertial particle dynamics, Phys. Rev. E 93 (2016) 033108.

[146] D. Garaboa-Paz, V. Perez-Munuzuri, A method to calculate finitetime Lyapunov exponents for inertial particles in incompressible flows, Nonlin. Proc. Geophys. 22 (2015) 571-577.

[147] B. Mosovsky, J. Meiss, Transport in transitory dynamical systems, SIAM J. Appl. Dyn. Sys. 10 (2011) 35-65.

[148] L. Duc, S. Siegmund, Hyperbolicity and invariant manifolds for planar nonautonomous systems on finite time intervals, Intern. J. Bifurc. Chaos 18 (2008) 641-674.

[149] M. Mathur, G. Haller, T. Peacock, J. Ruppert-Felsot, H. Swinney, Uncovering the Lagrangian skeleton of turbulence, Phys. Rev. Lett. 98 (2007) 144502.

[150] C. Senatore, S. Ross, Detection and characterization of transport barriers in complex flows via ridge extraction of the finite-time Lyapunov exponent field, Int. J. Numer. Meth. Engin. 86 (2011) 1163-1174.

[151] C. Mendoza, A. Mancho, The hidden geometry of ocean flows, Phys. Rev. Lett. 105 (2010) 038501.

[152] S. Balasuriya, A numerical scheme for computing stable and unstable manifolds in nonautonomous flows, Intern. J. Bifurc. Chaos 26 (2016) 1630041.

[153] G. Haller, F. Beron-Vera, Coherent Lagrangian vortices: the black holes of turbulence, J. Fluid Mech. 731 (2013) R4.

[154] C. Hirsch, Numerical Computations of Internal and External flows, 2nd Edition, Elsevier, Oxford, 2007. 
[155] F. Bottausci, I. Mezić, C. Meinhart, C. Cardonne, Mixing in the shear superposition micromixer: three-dimensional analysis, Proc. Trans. R. Soc. Lond. A 362 (2004) 1001-1018.

[156] F. Bottausci, C. Cardonne, C. Meinhart, I. Mezić, An ultrashort mixing length micromixer: the shear superposition micromixer, Lab Chip 7 (2007) 396-398.

[157] X. Niu, L. Liu, W. Wen, P. Sheng, Active microfluidic mixer chip, Appl. Phys. Lett. 88 (2006) 153508.

[158] P. Tabeling, M. Chabart, A. Dodge, C. Jullien, F. Okkels, Chaotic mixing in cross-channel micromixers, Phil. Trans. R. Soc. Lond. A 362 (2004) 987-1000.

[159] Y.-K. Lee, C. Shih, P. Tabeling, C.-M. Ho, Experimental study and nonlinear dynamic analysis of time-periodic micro chaotic mixers, J. Fluid Mech. 575 (2007) 425-448.

[160] S. Balasuriya, Dynamical systems techniques for enhancing microfluidic mixing, J. Micromech. Microeng. 25 (2015) 094005.

[161] S. Balasuriya, Optimal frequency for microfluidic mixing across a fluid interface, Phys. Rev. Lett. 105 (2010) 064501.

[162] S. Balasuriya, M. Finn, Energy constrained transport maximization across a fluid interface, Phys. Rev. Lett. 108 (2012) 244503.

[163] J. de Jong, R. Lammertink, M. Wessling, Membranes and microfluidics: a review, Lab Chip 6 (2006) 1125-1139.

[164] C.-H. Wang, G.-B. Lee, Automatic bio-sampling chips integrated with micro-pumps and micro-valves for disease detection, Biosensors Bioelectronics 21 (2005) 419-425.

[165] S. Balasuriya, Transport between two fluids across their mutual flow interface: the streakline approach, SIAM J. Appl. Dyn. Sys. 16 (2017) 1015-1044.

[166] P. Kloeden, E. Platen, H. Schurz, Numerical solution of SDE through computer experiments, Universitext, Springer-Verlag, Berlin, 1994. doi:10.1007/978-3-642-57913-4. 
Highlights for PHYSD_2017_213

- Comprehensively surveys Lagrangian Coherent Structure (LCS) methods

- Highlights that each LCS method provides different information, and has limitations

- Argues the need for generalizing LCS methods, and provides a general framework

- Provides examples from advecting and diffusing fluids, oceanography and microfluidics

- Discusses overarching issues relevant to LCSs and their generalizations 